\title{
Fast, multicolor 3-D imaging of brain organoids with a new single- objective two-photon virtual light-sheet microscope*
}

Irina Rakotoson, ${ }^{1,2,3,4+}$ Brigitte Delhomme, ${ }^{1,2,3+}$ Philippe Djian, ${ }^{1,2,3+}$ Andreas Deeg, ${ }^{5}$ Maia Brunstein, ${ }^{1,2,3}$ Christian Seebacher, ${ }^{5}$ Rainer Uhl, ${ }^{5}$ Clément Ricard, ${ }^{1,2,3, \S}$ and Martin Oheim ${ }^{1,2,3, \$, \varpi}$

${ }^{1}$ CNRS UMR 8118, Brain Physiology Laboratory, Paris F-75006, France

${ }^{2}$ Fédération de Recherche en Neurosciences CNRS FR 3636, Paris F-75006, France

${ }^{3}$ Faculté de Sciences Fondamentales et Biomédicales, Université Paris Descartes, PRES Sorbonne Paris Cité, F-75006 Paris, France

${ }^{4}$ Master programme: BCPP (Biologie cellulaire physiologie et pathologie), spécialité Neurosciences

${ }^{5}$ TILL.id, Am Klopferspitz 19, D-82153 Planegg/Martinsried, Munich, Germany

*corresponding author

Martin Oheim

martin.oheim@parisdescartes.fr

${ }^{+}$co-first authors

$\S$ co-last authors

*Elements of this work has been published in the Master thesis of I.R. [1]

The authors declare not conflict of interest. Rainer Uhl is the founder and owner of TILL.id, Andreas Deeg and Christian Seebacher are employees of TILL.id.

The funders of this study had no hands on the outcome or interpretation of the obtained results. 


\section{ABSTRACT}

48

49

Human inducible pluripotent stem cells (hiPSCs) hold a large potential for disease modeling. hiPSC-derived human astrocyte and neuronal cultures permit investigations of neural signaling pathways with subcellular resolution. Combinatorial cultures, and three-dimensional (3-D) embryonic bodies enlarge the scope of investigations to multi-cellular phenomena. A the highest level of complexity, brain organoids that - in many aspects - recapitulate anatomical and functional features of the developing brain permit the study of developmental and morphological aspects of human disease. An ideal microscope for 3-D tissue imaging at these different scales would combine features from both confocal laser-scanning and light-sheet microscopes: a micrometric optical sectioning capacity and sub-micrometric spatial resolution, a large field of view and high frame rate, and a low degree of invasiveness, i.e., ideally, a better photon efficiency than that of a confocal microscope. In the present work, we describe such an instrument that belongs to the class of two-photon (2P) light-sheet microsocpes. Its particularity is that - unlike existing two- or three-lens designs - it is using a single, low-magnification, high-numerical aperture objective for the generation and scanning of a virtual light sheet. The microscope builds on a modified Nipkow-Petráň spinning-disk scheme for achieving wide-field excitation. However, unlike the common Yokogawa design that uses a tandem disk, our concept combines micro lenses, dichroic mirrors and detection pinholes on a single disk. This design, advantageous for $2 \mathrm{P}$ excitation circumvents problems arising with the tandem disk from the large wavelength-difference between the infrared excitation light and visible fluorescence. 2P fluorescence excited in by the light sheet is collected by the same objective and imaged onto a fast sCMOS camera. We demonstrate three-dimensional imaging of TO-PRO3-stained embryonic bodies and of brain organoids, under control conditions and after rapid (partial) transparisation with triethanolamine and formamide (RTF) and compare the performance of our instrument to that of a confocal microscope having a similar numerical aperture. 2P-virtual light-sheet microscopy permits one order of magnitude faster imaging, affords less photobleaching and permits better depth penetration than a confocal microscope with similar spatial resolution. 


\section{INTRODUCTION}

The development of pharmacological treatments for neuropsychiatric and neurodegenerative diseases has been hampered by the poor availability of preclinical models that adequately capture the complexity of human disorders [2].

Human inducible pluripotent stem cells (hIPSCs) offer a promising platform for disease modeling and drug screening. A comparably new technique is the directed differentiation and reprogramming of patient fibroblasts into neurons, astrocytes, microglia and oligodendrocytes. Their combinational culture permits the growth of embryonic bodies (EBs) and brain organoids, 3-D cultures that - in many aspects - recapitulate the development of the human brain [3; 4]. Together, hIPSCs, EBs, and brain organoids enable observations and experiments that were previously inconceivable, neither on human subjects, nor in animal models $[5 ; 6]$. Recent reports of functional, fully vascularized brain organoids have spurred hopes of growing even larger 3-D cell assemblies [7], bringing the hitherto theoretical 'brain in a vat' within reach of the imaginable.

Elucidation of neural circuit (dys-)function would benefit from the detailed, 3-D visualization of the fine structure of neurons, astrocytes and blood vessels over large fields of view and deep in tissue. Large-scale neuroanatomical imaging has become possible in cleared tissue sections [8], brain organoids [9] or even entire brains [10], but in many cases the resolution is rather at level of cell bodies that at the synaptic scale. In addition to the difficulties associated with transparisation and tissue shrinking, imaging of large tissue volumes at spatial high-resolution presents considerable challenges: confocal and two-photon (2P) laser scanning microscopies set the 'gold-standard' for diffraction-limited fluorescence imaging, but - being in most of their implementations point-scanning, i.e., sequential techniques - the

\footnotetext{
${ }^{1}$ The 'brain in a vat' or 'brain in a jar' is a is a scenario used in a variety of Gedankenexperiments intended to draw out certain features of human conceptions of knowledge, reality, truth, mind, consciousness and meaning. It is an updated version of René Descartes' evil demon thought experiment. It has been extensively used by Hilary Putnam (Reason, Truth and History, 1981), in an argument inspired from Roald Dahl's short story, «William and Mary» (1959).
} 
101 image acquisition is often painstakingly slow. Particularly, the reconstruction of large

102 volumes often requires hours if not days of recording, putting high demands on mechanical 103 stability of the microscope, photostablity of the used fluorescent dyes, and incurring conside-

104 rable cost for beam time. Line- and multi-spot scanning schemes overcome these limitations

105 by parallelizing the excitation, but they trade off resolution against speed and they often have 106 relatively small fields of view, requiring image stitching for larger fields.

107 On the other end, selective-plane illumination microscopes (SPIM) [11] or light-sheet 108 microscopes [12] decouple fluorescence excitation and collection by using orthogonal 109 illumination and detection optical paths. Light-sheet microscopes have established themselves 110 as efficient workhorses for volume imaging in cleared tissue. It is the parallelization of both 111 excitation and fluorescence detection that allows for rapid 3-D imaging on these instruments $112[13 ; 14]$. However, one consequence of the lower-NA illumination and a result of excitation113 light scattering in not perfectly transparent samples, is that the axial resolution of light-sheet 114 microscopes remains poor compared to the optical sectioning achieved by spot-scanning 115 microscopes. Improvement has been made with 2P light-sheet excitation [15; 16], by 116 combining 2P-line excitation and confocal slit detection [17], by the use of Airy- [18] or

117 Bessel-beams for excitation [19;20;21], or a combination of these techniques [22; 23]. 118 However, many of these recent techniques are not yet commercial and they afford 119 considerable cost and complexity compared to standard 1- and 2P-laser scanning micro120 scopes.

121 Another limitation of light sheet microscopes results from their orthogonal arrangement of 122 excitation and collection objectives: the need for non-standard procedures for embedding and 123 holding the sample. Variants of light-sheet microscopes in which both illumination and 124 detection objectives are mounted at an oblique angle with respect to the tissue surface and the 125 sample half space is left free exist $[24 ; 25 ; 26]$, but they have remained comparably marginal. 
An ideal microscope for volume imaging in cleared brain tissue [27] would combine

speed, a sub- $\mu \mathrm{m}$ lateral and $\mu \mathrm{m}$-axial resolution, a mm-field of view, an excitation depth of a few $\mathrm{mm}$, a certain robustness to imperfect sample transparisation and a large free space under the objective.

Here, we present a microscope with excitation- and detection-parallelization that gets

131 close to this ideal by combining advantages of $2 \mathrm{P}$ laser-scanning and light-sheet techniques.

132 Our $\underline{\text { On}}$-axis 2-photon light-sheet generation in-vivo imaging system (OASIS) uses a vast 133 array of micro lenses arranged in four nested spirals on a single spinning disk to 134 simultaneously scan $~ 40$ independent excitation spots in the focal plane of a single, long135 working distance, low-magnification, high-NA objective [28]. Rotation of the disk at 5,000 136 rpm results in rapid multi-spot scanning and creates a virtual light sheet in the focal plane of 137 the objective. The fluorescence generated in each of the excitation spots is imaged through 138 the same objective onto a pinhole in the center of each micro lens. With the remainder of the 139 lens made opaque to (scattered) fluorescence by a dichroic coating sparing only the tiny 140 pinhole, only fluorescence emanating from the focus is detected. Each pinhole is imaged onto 141 a large-format scientific Complementary Metal Oxide Semiconductor (sCMOS) camera, 142 allowing near-diffraction limited imaging over a large field of view. This patented optical 143 design, combining micro-lenses and perforated dichroic mirrors on a single-spinning disk, 144 allowed us to retain the in-line, single-objective geometry of a classical microscope without 145 the requirement for orthogonal illumination. As a consequence, our OASIS microscope is 146 more versatile than two- or three-objective light-sheet microscopes. With its compact 147 footprint (43 $\mathrm{cm}$ by $12 \mathrm{~cm}$, or $17 " \times 5$ "), it can accommodate large samples (cells, slices, 148 explants and entire animals, in vivo) without requiring tedious mounting procedures or special 149 sample holders. The OASIS concept combines the optical sectioning, spatial resolution and 150 field-of-view of a 2P-scanning microscope with the speed of a light-sheet microscope. Due to 
bioRxiv preprint doi: https://doi.org/10.1101/461335; this version posted November 3, 2018. The copyright holder for this preprint (which was not certified by peer review) is the author/funder. All rights reserved. No reuse allowed without permission.

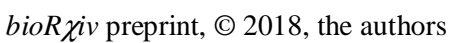

Rakotoson, Delhomme, Djian et al., (2018)

151 2P excitation, out-of-focus fluorescence excitation, photo-bleaching and photo-damage are

152 much reduced compared to a classical confocal microscope. We here describe this new 153 microscope and compare it to a confocal laser-scanning microscope (CLSM) for imaging 154 clarified brain organoids with nuclear staining.

155

156

157

158

159

160

161

162

163

164

165

166

167

168

169

170

171

172

173

174

175

176

177

178

\section{RESULTS AND DISCUSSION}

\section{Wide-field two-photon microscopy at diffraction-limited resolution}

With our $\underline{\text { On}}$-axxis 2-photon light-shheet generation in-vivo imaging system (OASIS), we retain the in-line geometry of a classical upright microscope with a single objective lens. We introduce a novel spinning-disk concept, rethought and specifically designed for wide-field $2 \mathrm{P}$ microscopy, Fig. 1A. Briefly, the expanded and shaped beam of a fs-pulsed infrared laser is focused by an array of micro lenses to produce some 40 evenly lit excitation spots. These spots are imaged by the tube lens and objective into the sample plane where they are each spaced, on average, by $28 \mu \mathrm{m}$, Fig. 1A, inset $\mathbf{0}$. A total of almost 5,000 micro lenses are arranged in four nested spirals that scan these spots upon rotation of the disk. At 5,000 rpm (i.e., one turn every $12 \mathrm{~ms}$, much shorter than camera integration times used here) the multispot scanning generates a virtual light sheet permitting wide-field, direct-view 2P imaging. Unlike earlier 2P-spinning disk microscopes $[29 ; 30 ; 31]$ that were based on modified Yokogawa-type spinning-disk confocal microscopes, we use a different disk geometry. Where the Yokogawa dual-disk design requires two disks, one with micro lenses, the other one with confocal pinholes, the OASIS microscope relies on a single disk on which micro lenses and pinholes that are arranged on the different faces of the same optical element. Specifically, we do not use an extra dichroic, but each micro lens comes with its own dichroic mirror; the dielectric coating is omitted over a central circular aperture of $60-\mu \mathrm{m}$ diameter. The use of a single disk is advantageous in view of the large wavelength difference between the near- 
179 infrared excitation and the visible fluorescence, which had previously reduced the efficiency

180 of 2P-spinning disk miroscopes. However, this simplification comes at a price as it required a

181 complete re-design of the microscope optical path. On the excitation side, the micro lenses

182 must be uniformly illuminated with a collimated fs-pulsed IR laser beam to generate an array

183 of focused spots. On the collection side, to pass the pinholes, the detected fluorescence must

184 arrive focused at the level of the pinholes. The required optical path-length difference be-

185 tween excitation and emission light is achieved by introducing a corrective distance element

186 in the non-infinity space: while the longer-wavelength excitation light passes straight through

187 the device, the shorter-wavelength fluorescence takes a detour and travels a longer path to

188 produce the desired focal offset, inset 2. This patented optical scheme critically relies

189 extremely flat shallow-incidence long-pass dichroic mirrors to preserve the phase front of the

190 beam and maintain the optical resolution.

191 The 2P-excited fluorescence generated in each of the spots is collected through the

192 same objective and imaged onto the tiny pinholes in the dichroic coating of the microlenss

193 (Fig. 1A, inset 2). Relay optics then images these pinholes onto a large-format sCMOS

194 camera. The resulting pixel size in the sample plane is $182 \mathrm{~nm}$, within the Nyquist limit.

195 This detection is partially confocal (the $60-\mu \mathrm{m}$ pinhole diameter correspond to a con-

196 focal aperture of 2 Airy units), so that only ballistic and snake-like photons but not scattered

197 fluorescence contribute to the signal, as illustrated by the exponential fluorescence drop with

198 increasing sample turbidity. Yet, as a result of the longer wavelength of excitation light, the

199 signal drop observed with the OASIS microscope was only half of that observed with a 1-P

200 CLSM at 633-nm excitation, Fig. $1 B$. We can attribute the improved depth penetration of the

201 OASIS uniquely to excitation effects, because stopping down the confocal pinhole from 2 to 1

202 Airy units did not measurably alter the fluorescence decay on the CLSM. 
With its small footprint (43 $\mathrm{cm}$ by $12 \mathrm{~cm}$ ) and $22-\mathrm{cm}$ clearance under the objective, the OASIS microscope offers facile access and ample space around the objective, making it an ideal platform for imaging large samples, but also for placing electrodes, application dual-color detection by way of a custom image splitter, Fig. S1.

With the $\times 4.6$ beam expander and a $\times 25 / \mathrm{NA} 1.1 \mathrm{w}$ low-mag high numerical aperture 210 (NA) dipping objective [28], the OASIS microscope features a field of view with a $200 \mu \mathrm{m}$ image diagonal. A 5- to 6-fold larger field, making full use of the nominal field-of-view of the same objective would be possible, but it requires more micro-lenses to be illuminated, which in turn requires a more powerful laser than ours. frame rate, a large field of view and it resolves tiny subcellular detail. We illustrate the submicrometric resolution by imaging the fine tip of a spine from an autofluorescent pollen grain, a typical test sample for 2P-microscopes, Fig. $1 D$.

For estimating the optical sectioning capability of our OASIS microscope, we recorded the axial (z-) intensity profile from a green fluorescent Chroma test slide and we quanti-

220 fied the $z$-resolution $(\Delta z)$ by the full-width at half maximum (FWHM) of a Gaussian fitted 221 with the derivative of the $z$-profile [32], Fig. 1E. Repeating the same experiment on a ZEISS 222 LSM710 demosntrates that OASIS offered a 1.3-fold better optical sectioning than the 223 confocal laser-scanning microscope (with the pinhole diameter set to 2 Airy diameter and with a dipping objective having a similar NA, $1.1 \mathrm{w}$ vs. $1.0 \mathrm{w} ; \Delta z_{\mathrm{OASIS}}=2.75 \pm 0.02 \mu \mathrm{m}$ vs.

$\left.225 \Delta z_{\text {CLSM, } 2 \text { Airy }}=3.47 \pm 0.02 \mu \mathrm{m}\right)$. In fact, its optical sectioning is close to that of a CLSM with 226 the pinhole stopped down to 1 Airy diameter $\left(\Delta z_{\mathrm{CLSM}}, 1\right.$ Airy $\left.=2.55 \pm 0.02 \mu \mathrm{m}\right)$, Fig. S2. 
The acquisition speed will be in practice be limited by the available signal, but the

228 theoretical minimal exposure time is bounded by the Nyquist limit, i.e., the time required for 229 two full rotations of the spiral on the disk. With a rotation time of $12 \mathrm{~ms}$ at $5,000 \mathrm{rpm}$ and 230 four nested spirals, the minimal theoretical exposure time is $3 \mathrm{~ms}$, i.e., $6 \mathrm{~ms}$ when taking into 231 account Nyquist's sampling theorem. Integration times should be multiples of $6 \mathrm{~ms}$ for 232 obtaining a homogeneously lit field of view. With the 100-fs pulses and $\mathrm{mW}$ average laser 233 power, $\langle P\rangle$, per illumination spot used here, typical exposure times were of the order of 234 hundred ms, full frame, more than one order of magnitude faster than typical time required a 235 similarly resolved image with a $2 \mathrm{P}$-scanning microscope.

Taken together, our OASIS virtual 2P light-sheet microscope offers better depth pene-

tration, a similar if not better spatial resolution and a considerably higher speed than a conventional CSLM.

\section{RTF-cleared TO-PRO3-labeled embryos as a test sample for 1- and 2P microscopies}

245 Next, we evaluated the performance of the OASIS microscope for 3-D imaging of partially

246 cleared brain tissue. We sought for a stereotypic, sparsely but homogenously labeled and thick

247 sample. To allow for a direct comparison between 1P-CLSM and our 2P virtual-light sheet

248 microscope, this labeling needed to be suitable for both linear- and non-linear excitation. To

249 minimize scattering and improve the depth penetration, we searched for a red-exciting, deep-

250 red emitting fluorophore. With these constraints in mind we opted for the nuclear stains TO-

251 PRO3 and Methyl Green (MG), having 1P-fluorescence excitation/emission maxima of $252642 / 661 \mathrm{~nm}[33])$ and 632/650 nm [34], respectively.

253 During preliminary experiments in $7-\mu \mathrm{m}$ thin sections of a fixed (E14.5) mouse 254 embryo, we found TO-PRO3 fluorescence to be 2.1-fold brighter than that of MG upon 633- 
$255 \mathrm{~nm}$ excitation. We observed an even larger intensity ratio $(\times 5.5)$ upon $2 \mathrm{P}$-excitation at 760

$256 \mathrm{~nm}$, Fig. 2A. Although non-linear excitation of TO-PRO3 at 1,100 nm has been reported [35;

$25736]$ we measured the $2 \mathrm{P}$-action spectra and found peak excitations at 760 and $750 \mathrm{~nm}$ for TO-

258 PRO3 and MG, respectively, Fig. 2B. Compared to the reported 1,100-nm excitation these 259 shorter wavelengths are within the tuning range of the standard Ti:Saphh laser and they 260 minimize thermal damage from near-infrared absorption and focal heating [37], a particular 261 concern for the multi-spot excitation scheme used here.

262 We next optimized the tissue transparisation procedure. Among the available methods 263 (see [38] for review), we focused on TDE [39], Clear ${ }^{\mathrm{T} 2}$ [40] and RTF clearing [41]. The 264 rationale was that these methods require only short clearing episodes and they use solvents 265 compatible with dipping objectives. Mouse embryos were most transparent with TDE (60\%), 266 followed by RTF and, by far, Clear ${ }^{\mathrm{T} 2}$, for which the tissue was even more opaque than the 267 non-cleared control (probably due to volume shrinkage), Fig. $2 C$.

268 In clearing, transparency is one issue, fluorescence preservation another. Depending 269 on the very method used, the observed signal loss was dramatic, with a $99.9 \%$ and $92 \%$ 270 attenuation of TO-PRO3 fluorescence following Clear ${ }^{\mathrm{T} 2}$ and RTF clearing, respectively, Fig. $2712 D$. Increasing the laser power by a factor of 20 allowed us to acquire confocal images of TO272 PRO3 stained nuclei in slices of RTF-cleared embryos, Fig. 2E, whereas TDE clearing 273 attenuated the fluorescence to undetectable levels, Fig. S3. To develop an order-of-magnitude 274 idea of the laser powers required for obtaining similar signal-to-noise levels with the OASIS 275 and CSLM, we finally compared images acquired upon 1- (at $633 \mathrm{~nm}$ ) and 2-excitation (at $276760 \mathrm{~nm}$ ) of TO-PRO3 labeled nuclei in a thin section of RTF cleared mouse embryo. With a 277 confocal aperture of 2 Airy, we found a factor of $\times 4,000$ btween linear and non-linear 278 excitation $(2 \mu \mathrm{W}$ vs. $8 \mathrm{~mW} / \mathrm{spot}$, respectively). 
bioRxiv preprint doi: https://doi.org/10.1101/461335; this version posted November 3, 2018. The copyright holder for this preprint (which was not certified by peer review) is the author/funder. All rights reserved. No reuse allowed without permission.

bioR Xiv preprint, (C) 2018, the authors

Rakotoson, Delhomme, Djian et al., (2018)

Based on these results, we decided to combine TO-PRO3 nuclear staining and RTF clearing for directly comparing the performance of confocal and OASIS microscopes.

281

282

283

284

285

286

287

288

289

290

291

292

293

294

295

296

297

298

299

300

301

302

303

304

305

306

307

(figure 2 close to here)

\section{Faster and less invasive acquisition of 3-D image stacks}

Tracing fluorescent dendrites and axons to study the projections and connectivity in small cellular net-works is a major goal of current neuroanatomy. At 250-nm lateral and micrometric axial sampling and with typical pixel dwell times of $1(10 \mu \mathrm{s})$, sequential singlespot scanning schemes are necessarily slow, requiring $4(40) \mathrm{ms}, 4(40) \mathrm{s}$ and more than 1 (10) hrs for the acquisition of 3-D image stacks from cubes of $10 \mu \mathrm{m}, 100 \mu \mathrm{m}$ and $1 \mathrm{~mm}$ sidelength, respectively. Parallelizing both the excitation and emission detection, as with our OASIS microscope is expected to considerably speed up the imaging of such large data sets. Using TO-PRO3 nuclear staining as a proxy, we acquired $z$-stacks of images in RTFcleared mouse embryos. Comparing the achievable imaging depths with the OASIS microscope in non-cleared (30 $\mu \mathrm{m}, \mathbf{F i g} . \mathbf{3} A$ and $\mathbf{S 5} A) v s$. RTF-cleared samples $(90 \mu \mathrm{m}, \mathbf{F i g} . \mathbf{3} B$ and S5A) we noted the 3-fold larger attainable imaging depth and well-preserved structural details. Next, we evaluated the photobleaching with the OASIS and confocal microscope. We first compared 2P-light sheet and confocal acquisition at shallow imaging depths, by continuously recording images of the same region of interest (ROI) at $30-\mu \mathrm{m}$ depth in an non-cleared embryo. At the same initial signal-to-noise ratio for both microscopes, We observed a $\sim 3 \%$ intensity loss after the first image with the OASIS microscope $\left(t_{\exp }=480 \mathrm{~ms}\right.$ per image, 15.7 $\mathrm{mW} / \mathrm{spot}$ for the OASIS), whereas the signal remained relatively stable after a confocal scan. Fitting a monoexponential with the OASIS bleaching data revealed a 1/e (37\%) loss of fluorescence every 21 frames, Fig. 3C. Thus, fs-pulsed non-linear excitation results in a significantly higher pleaching in superficial tissue layers. However, for 3-D imaging of 
308 thicker sections, confocal microscopy rapidly produced much faster photobleaching than the

309 OASIS because it required higher and higher laser powers to maintain image contrast at 310 greater imaging depths; in fact, acquiring the first complete $z$-stack with the CSLM attenuated

311 the TO-PRO-3 fluorescence so much that a second acquisition was impossible, Fig. $3 D$.

312 We attribute the much higher volume photobleaching upon $1 \mathrm{P}$ confocal imaging in 313 non-cleared embryos to four reasons, $(i)$, tissue scattering at $633 \mathrm{~nm}$ was roughly double that 314 of near-infrared light. The exponential scattering losses of excitation photons must be 315 compensated for by exponentially increasing the excitation powers with increasing imaging 316 depth; (ii), as a consequence of linear (1P) excitation, off-focus excitation of fluorophores 317 located above and below the imaged plane causes bleaching, too, i.e., at any plane, bleaching 318 occurs throughout the entire tissue volume while only one plane is imaged; (iii), although not 319 contributing to imaging, the scattered 1P excitation light neverthelass excites (out-of-focus) 320 fluorescence, which - in addition to the ballistic out-of-focus excitation - additionally 321 contributes to photobleaching. Non-linear (2P) excitation, on the other hand, confines both 322 fluorescence excitation and photobleaching to the focal plane, with the result fo better 323 preserving the sample outside the plane which is actually imaged; (iv), image acquisition was 324 4-times faster on the OASIS compared to confocal scans (480 ms/image vs. 1.815 s/image for 325 a similar image contrast), reducing the overall exposure of the sample.

326 We note that he better performance of the OASIS concept comes essentially from the 327 excitation side, because with a confocal pinhole of 2 Airy, on the fluorescence collection side, 328 both instruments should perform similarly. Finally, not only taking into account the loss of 329 signal, but also that of Weber contrast, we found a three-fold larger effective depth pene330 tration for the OASIS microcope, Fig. S5A.

331 Taken together, the OASIS microscope combines the advantages of $2 \mathrm{P}$-exciation and 332 wide-field imaging. Compared to the 1P-confocal, it achieves higher $z$-resolution, affords less 
333 photobleaching in 3-D samples and considerably speeds up data acquisition, thus allowing a 334 more efficient and less invasive volume imaging.

335

336

337

338

340

341

344

350

351

352

354

(figure 3 close to here)

\section{Fast volume acquisition from mouse embryonic bodies and brain organoids}

We continued our comparison by imaging day-7 embryonic bodies (EB), Fig. 4A, again stained with TO-PRO3. Images acquired at different depths displayed rounded structures with lumina inside tissue and revealed strong mitotic activity, Fig. 4B. The rounded structures presumably correspond to neuroepithelial-like structures that are readily formed within EBs, indicating the inherent ability of the ectoderm to differentiate into neural lineages [42]. The sub- $\mu \mathrm{m}$ resolution of the OASIS microscope allowed us the detailed characterization of the different mitotic figures throughout the entire $160-\mu$ m thickness of the EB, Fig. $4 C$.

Acquirig a 3-D image stack at a $z$-spacing of $0.5 \mu \mathrm{m}$ (Fig. S5B) allowed us reconstructing complete EBs and realizing high-resolution projections along the orthogonal axes, Fig. 4D, revealing fine structural detail and including again mitosis across its entire volume, Fig. 4E. Volume imaging of entire EBs was almost 4-times faster with the OASIS compared to the CLSM (a 200- $\mu \mathrm{m} z$-stack with a $0.5 \mu \mathrm{m}$ z-spacing required 3'12" vs. 12'6"), with no detectable photobleaching. Together, these features make the OASIS microscope an ideal setup for 3-D the detailed characterization of EB development.

\section{(figure 4 close to here)}

Similar if not larger $z$-stacks were acquired a from RTF-cleared day-11 brain organoids, Fig. 5A. At this early developmental stage, the neuroepithelium has been induced and forms buds that undergo 3-D growth within the Matrigel droplets [43]. Our observations highlight strong morphological modifications during this tissue expansion. A recurrent feature was that TO- 
359 PRO-3 labeled nuclei were rounded just below the surface of the brain organoids, whereas 360 polymorph and diamond-shapes prevailed at greater imaging depths, Fig. $\mathbf{5} B$. Also, the cell 361 density and nuclear labeling changed markedly with depth. Orthogonal planes revealed 362 compact groups of nuclei with stronger fluorescence, Fig. $\mathbf{5} C$, as well as cavities and rounded 363 structures with a neuroepithelium-like shape. As before in EBs, the resolution of the OASIS 364 microscope allowed us to detect the presence of mitotic figures at the luminal side, Fig. $\mathbf{5} D$. In 365 brain organoids, typical achievable imaging depths were around $200 \mu \mathrm{m}$, reflecting the 366 densification and opacification of the tissue during the development of an EB towards a brain 367 organoid.

368 With its large field-of-view, increased depth penetration, low photobleaching and greater speed of acquisition compared to the CSLM, the OASIS optical scheme lends itself ideally to the observation of entire EBs and whole brain organoids. Thin sections can be studied at depth with sub-cellular ( $\mu \mathrm{m}-)$ resolution, without mechanical slicing. The reduced

372 complexity compared to a classical light-sheet microscope, its compact mono-block design 373 and comparble ease-of-use make it an ideal companion for functional neuroanatomy. The ongoing integration of a compact, fixed-wavelength high-power fs-pulsed laser into this package will make the OASIS a unique, portable, alignment-free bench-top 2P-microcope.

376

377

378

379

380

381

382

383

384

385

\section{(figure 5 close to here)}

\section{CONCLUSION}

Work on brain organoids offers several distinctive advantages over classical disease models: (i), derived from patient fibroblasts, they raise less concerns than animal experimentation and work on human explants (see, however, [44] for an emerging awareness of the ethical issues associated with these 3-D cultures); (ii), they avoid the limitations of animal models that are often only a poor proxy of human pathology; (iii), they allow studies of rare or sporadic cases, 
bioRxiv preprint doi: https://doi.org/10.1101/461335; this version posted November 3, 2018. The copyright holder for this preprint (which was not certified by peer review) is the author/funder. All rights reserved. No reuse allowed without permission.

bioR Xiv preprint, () 2018, the authors

Rakotoson, Delhomme, Djian et al., (2018)

388 for which genetic models are missing; (iv), they allow observing the onset of the disease

389 during the early steps of the brain development, opening opportunities for studies that would

390 be impractical or inacceptable on human embryos or infants.

391 For this field of applications, the OASIS 2P virtual light-sheet microscope is a

392 compact, versatile and cost-efficient 2P wide-field research instrument allowing imaging of

393 hiPSC cultures, embryonic bodies and brain organoids.

394

395

396

397

398

399

400

401

402

403

404

405

406

407

408

409

410

411

412

413

414

415

416

417

418

419

420

421

422

423

424

425

426

427

428

429

430

431

\section{MATERIALS AND METHODS}

\section{Ethics statement}

All experimental procedures were performed in accordance with the French legislation and in compliance with the European Community Council Directive of November 24, 1986 (86/609/EEC) for the care and use of laboratory animals. The used protocols were approved by the local ethics committee.

\section{Sample preparation}

hiPSC culture and formation of embryonic bodies. Episomal human induced pluripotent stem cells (hIPSCs, Gibco) were cultivated on mitomycin-treated mouse embryonic fibroblasts using DMEM/F12 medium (Invitrogen), supplemented with 10\% knockout serum (Gibco). When hIPSCs had reached about $80 \%$ confluence, they were detached with versene (ThermoFisher). Cell aggregates were removed and a single-cell suspension obtained with a cell strainer having a $100-\mu \mathrm{m}$ mesh size (Corning). For the formation of embryoid bodies (EBs), $10^{4}$ cells were inoculated in $100 \mu \mathrm{l}$ in each well of a ultra-low attachment, roundbottomed 96-well plate (Corning) and cultivated during 9 days in EB formation medium (StemCell Technologies).

Mouse embryos. Embryos were age E10.5 and E14.5. Mice were killed by cervical dislocation, the abdominal cavity was opened and the uterine horns were removed. Embryos were collected under a macroscope (Nikon SMZ800) and immersed in formalin (buffered $10 \%$ formaldehyde, VWR) overnight. They then were stored at $4{ }^{\circ} \mathrm{C}$ in PBS / Sodium Azide 0,02\%. E10.5 embryos were used for whole-embryo transparisation; E14.5 embryos were embedded in Optimal Cutting Temperature (OCT) compound and sliced into 7- $\mu$ m-thin sections on a cryotome (Cryocut 1800, Leica). 
bioRxiv preprint doi: https://doi.org/10.1101/461335; this version posted November 3, 2018. The copyright holder for this preprint (which was not certified by peer review) is the author/funder. All rights reserved. No reuse allowed without permission.

bioR Xiv preprint, (C) 2018, the authors

Rakotoson, Delhomme, Djian et al., (2018)

\section{Staining, clearing and embedding}

433

434

435

436

437

438

439

440

441

442

443

444

445

446

447

448

449

450

451

452

453

454

455

456

457

458

459

460

461

462

463

464

465

466

467

468

469

470

471

472

473

474

475

476

477

478

479

480

481

Nuclear staining. Samples were permeabilized by a $0.2 \%$ TritonX100 solution in PBS (during $15 \mathrm{~min}$ for $7-\mu \mathrm{m}$-thin embryo slices and EBs, $20 \mathrm{~min}$ for brain organoids, $1 \mathrm{~h}$ for the wholemount E10.5 embryo). They were then incubated overnight in a 1:1000 solution of TO-PRO3 (Invitrogen) or of chloroform-purified Methyl Green (Merck) in PBS and finally washed in PBS. At this point, non-cleared samples were mounted in a PBS-filled chamber under a glass coverslip for microscopy. We used a home-made recording chamber, that was modified from chambers designed for imaging and available as 3-D printer templates: https://idisco.info/idisco-protocol/).

Clearing. After nuclear staining, samples were processed for one of the three following clearing methods: TDE [39], Clear ${ }^{\mathrm{T} 2}$ [40] or RTF [41].

TDE. Samples were successively immersed in increasing concentrations $(20 \%, 40 \%$ and $60 \%)$ of TDE (Sigma) solutions in PBS. The duration of each incubation varied as a function of the sample size: $1 \mathrm{~h}$ for $7-\mu \mathrm{m}$ embryo slices and for EBs, $3 \mathrm{~h}$ for a whole E10.5 embryo.

Clear $^{T 2}$. Samples were immersed successively in, (i), a $25 \%$ formamide (Sigma) / $10 \%$ polyethylene glycol (PEG8000, Merck) solution in PBS (10 min for embryo slices and embryonic bodies, 30 min for a whole E10.5 embryo); (ii), a 50\% formamide / 20\% PEG8000 solution in PBS (5 min for slices and EBs, 15 min for whole embryo); (iii), a 50\% formamide / 20\% PEG8000 solution in PBS (1h for slices and for EBs, 3h for a whole E10.5 embryo).

RTF. Samples were successively immersed in, (i) a 30\% triethanolamine (TEA, Sigma) / 40\% formamide (Sigma) solution (15 min for slices and for embryonic bodies, $20 \mathrm{~min}$ for brain organoids, 3h20min for a whole E10.5 embryo); (ii) a 60\% TEA / 25\% formamide solution ( $25 \mathrm{~min}$ for slices and for embryonic bodies, $30 \mathrm{~min}$ for brain organoids, $5 \mathrm{~h}$ for embryo); (iii) a $70 \%$ TEA / 15\% formamide solution ( $25 \mathrm{~min}$ for slices and for EBs, $30 \mathrm{~min}$ for brain organoids, $5 \mathrm{~h}$ for whole embryo). In either case, after clearing, samples were mounted under a glass coverslip in a chamber filled with the respective final solution.

\section{Microscopy}

Confocal microscopy. We used a Zeiss LSM 710 microscope with a $\times 63 / 1.4 \mathrm{NA}$ oilimmersion objective, for the acquisition of the confocal data shown in figs. 2 and S3, and a $\times 40 / 1.0 \mathrm{NA}$ water-immersion objective for tissue sections and thick samples (embryo, EB, brain organoid), respectively. For a fair comparison of the performance for imaging thick samples of the confocal and 2P-virtual light sheet OASIS microscope, we set the confocal pinhole to 2 Airy units and the scanned area was restricted to $900 \times 900$ pixels with a zoom resulting in an effective pixel size of $0.182 \mu \mathrm{m}$, equivalent to the OASIS. We also acquired confocal images at 1 Airy for comparison. The laser powers delivered to the sample are indicated in each figure. For 1PEF, we excited TO-PRO3 using the 633-nm line of a HeNe gas laser (Lasos). Fluorescence was collected in between $646 \mathrm{~nm}$ and $725 \mathrm{~nm}$.

$2 P$-virtual light-sheet microscopy. The optical path of OASIS microscope is schematized in Fig.1. A detailed technical characterization is given in a companion paper, (Ricard, Deeg, et al., submitted). Briefly, 2P fluorescence is excited in 40 foci. These spots are scanned across the sample and the fluorescence emission from the spots confocally detected, similar to a 
bioRxiv preprint doi: https://doi.org/10.1101/461335; this version posted November 3, 2018. The copyright holder for this preprint (which was not certified by peer review) is the author/funder. All rights reserved. No reuse allowed without permission.

bioR Xiv preprint, (C) 2018, the authors

Rakotoson, Delhomme, Djian et al., (2018)

482 Nipkow-Petráň spinning-disk microscope [45]. However, for optimal 2P excitation, a disk 483 was manufactured that combines micro-lenses, dielectric long-pass (LP) coating and confocal 484 pinholes. On the front side of this glass disk (borofloat B33, 100-mm diameter, 2-mm thick) $485>5,000$ micro-lenses are arranged in four nested spirals were micro-machined (diameter $D=$ $486666 \mu \mathrm{m}$, focal length $f=7.8 \mathrm{~mm}$, lens-area-fill factor: $73.2 \%$, the average inter-lens spacing is 487 about $666 \mu \mathrm{m})$. The dielectric coating $\left(T_{\text {ave }}<0.05 \%\right.$ for $400-675 \mathrm{~nm}, T_{\text {ave }}>93 \%$ for $730-1100$

488

489

490

491

492

493

494

495

496

497

498

499

500

501

502

503

504

505

506

507

508

509

510

511

512

513

514

515

516

517

518

519

520

521

522

523

524

525

526

527

528

529

530

531

$\mathrm{nm}$ ) on the rear side has pinholes (i.e. non-coted areas, each having $60-\mu \mathrm{m}$ diameter) centered on the micro-lenses on the front side of the disk (see Fig.1, inset).

For 2P excitation, the Gaussian beam of a fs-pulsed Ti:Saph laser (Spectra-Physics MaiTai HP with DeepSee ${ }^{\mathrm{TM}}$ module) was shuttered (AOM: AA Opoelectronic), expanded and collimated to a beam diameter of about $5 \mathrm{~mm}\left(1 / e^{2}\right)$ and aligned on the rear side of the disk. The disk transmits the IR light and the microlenses on the front side generate a spiral pattern of foci $7.8 \mathrm{~mm}$ in front of the disk. This pattern is imaged onto the sample by a telescope formed by the tube lens $(f=200 \mathrm{~mm})$ and water-immersion objective (Nikon CFI75 Apochromat LWD 25× MP, NA 1.1w).

2PEF is detected through the same objective and tube lens. However, between the tube lens and the disk, two LP dichroics (custom design and manufacture from Alluxa, 50\%-cut-on at $705 \mathrm{~nm}, T_{\text {ave }}<1.5 \%$ from $400-695 \mathrm{~nm}, T_{\text {ave }}>96 \%$ from $715-1100 \mathrm{~nm}$, flatness $<0.25$ wave P-V per inch, $d=1 \mathrm{~mm}$ ) were used at $18^{\circ}$ angle of incidence (AOI) to image the emission from the excitation spots onto the pinholes of the disk. As a consequence, the collected fluorescence from the excitation spots arrives focused at the level of the pinholes whereas out-of-focus fluorescence and scattered emission light is blocked. The intermediate image in the pinhole plane is separated from the IR excitation light by an AOI $45^{\circ}$ primary dichroic LP filter (Custom design, manufactured by Alluxa, AOI $45^{\circ}$, flatness $<0.25$ wave P$\mathrm{V}$ per inch, $d=1 \mathrm{~mm}$ ) and magnified by a $f_{1}=140 \mathrm{~mm}$ and $f_{2}=200 \mathrm{~mm}$ telescope onto an sCMOS camera (PCO.edge 4.2, chip size $13.3 \mathrm{~mm}$ x $13.3 \mathrm{~mm}$ ). Due to this magnification, the image size on the chip is about $7.1 \mathrm{~mm}$ and the total magnification of the system is $\times 36$. To block residual IR excitation light, a multi-photon-emitter (ET700SP-2P, Chroma) was used. For dual-color imaging a LP dichroic under AOI $15^{\circ}$ (in fact a Semrock 532-nm laser BrightLine Di02-R532, AOI $=45^{\circ}$. For AOI $=15^{\circ}$ its edge shifts to $568 \mathrm{~nm}$ ) was used in combination with a knife-edge right-angle prism to split the image in the non-infinity space between the $f=200-\mathrm{mm}$ lens and the camera chip. The red emission $(>568 \mathrm{~nm})$ is imaged on one half of the sCMOS chip, and the green $(<568 \mathrm{~nm})$ on the other, without further emission filtering. The fluorescence signal at the margin of the field of view is relatively weak, and cropping the image of about 7.1-mm diameter to the half chip size of $6.65 \mathrm{~mm} \times 13.3 \mathrm{~mm}$ is justified. Image cropping, rotation and alignment were automatized in a custom-written macro in FIJI [46].

The upright microscope body was constructed as a rugged monocoque, machined from a single metal block (see photos), with slots for the optical and opto-mechanical elements at pre-defined places. A highly folded realization of the beam-path optimized by ZEMAX allowed the compact design. We used a voice-coil based $z$-objective drive, combining accuracy and precision over a wide focusing range. A flipable mirror was used to toggle between 2P-imaging and trans- or reflected-light imaging. This alternative beam path uses a different $f$ $=140$-mm tube lens in combination with a small USB camera (Point Grey, BFLY-U323S6M-C). Having an effective magnification of $\times 17.5$ this imaging mode was used for getting an overview of the sample and for identifying regions of interest (ROIs) to be imaged in $2 \mathrm{P}$ fluorescence. All image acquisition parameters, the laser and the objective focus drive 
bioRxiv preprint doi: https://doi.org/10.1101/461335; this version posted November 3, 2018. The copyright holder for this preprint (which was not certified by peer review) is the author/funder. All rights reserved. No reuse allowed without permission.

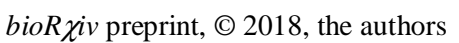

Rakotoson, Delhomme, Djian et al., (2018)

532

533

534

535

536

537

538

539

540

541

542

543

544

545

546

547

548

549

550

551

552

553

554

555

556

557

558

559

were controlled through an in-house microcontroller imaging software. The used excitation wavelength $\lambda_{\mathrm{ex}}$, laser power $\langle P\rangle$, and z-spacing $\Delta z$ for stacks were software controlled and are specified in the figure legends. Images were acquired without software binning at the full sCMOS resolution.

\section{Image analysis and quantification}

Images were analyzed and displayed using FIJI. For a better visibility of the faint fluorescent signals at greater imaging depths, we used a nonlinear look-up table $(\gamma=0.6)$ for Figs. 4B-E and 5C-D.

Weber contrast was calculated as $C_{\mathrm{w}}=(F-B) / B$, where $F$ and $B$ are the fluorescence intensities of the image and background, respectively. $B$ was measured as the mean intensity of a cellfree region and $F$ corresponded to the mean intensity in a small ROI $(18 \mu \mathrm{m} \times 18 \mu \mathrm{m})$ in the center of the image for fig. S5A, and to the whole image in fig. S5B. (In fig. S5A the sample was inclined respect to the optical axis so that only a small region could be used to quantify the penetration depth).

\section{Statistics}

Results are at least triplicates of three experiments and are represented as mean \pm SD. Student's $t$-test was used to compare among experiments. Data were processed and figures prepared using IGOR Pro (Wavemetrics). 
bioRxiv preprint doi: https://doi.org/10.1101/461335; this version posted November 3, 2018. The copyright holder for this preprint (which was not certified by peer review) is the author/funder. All rights reserved. No reuse allowed without permission.

bioR Xiv preprint, (C) 2018, the authors

Rakotoson, Delhomme, Djian et al., (2018)

560 List of abbreviations

561

562

$2 \mathrm{P}$

- two-photon

563

564

565

566

567

568

569

570

571

572

573

574

575

576

577

578

579

580

581

582

583

584

585

586

587

588

589

590

591

592

593

594

595

596

597

598

599

600

601

602

603

604

605

606

607

608

3-D - $\quad$ three-dimensional

AOI - - angle of incidence

BSA

CLSM

DMEM

EB

FWHM

bovine serum albumin

hiPSC - $\quad$ human inducible pluripotent stem cell

LP

- $\quad$ long-pass (filter)

MG

- $\quad$ Magnesium Green

NA

- numerical aperture

OASIS - $\quad$ On-ạxis 2-photon light-ṣheet generation in-vivo inmaging system

OCT $\quad-\quad$ opimal cutting temperature

PBS - $\quad$ phosphate-buffered solution

PEG - - polyethylene glycol

RI $\quad-\quad$ refractive index

ROI

- $\quad$ region of interest

RTF

- $\quad$ Rapid clearing method based on Triethanolamine and Formamide

sCMOS - $\quad$ scientific Complementary Metal Oxide Semiconductor

SD $\quad-\quad$ standard deviation

SPIM - $\quad$ selective-plane illumination

TDE - 2,2'-thiodiethanol

TO-PRO-3 - $\quad$ a carbocyanine monomer nucleic acid stain with red excitation and farred fluorescence $(642 \mathrm{~nm} / 661 \mathrm{~nm})$ similar to Alexa Fluor 647 or Cy5. It 609 is among the highest-sensitivity probes for nucleic acid detection. 


\section{Author contributions}

611

612

613

614

615

616

617

618

619

620

621

622

623

624

625

626

627

628

629

630

631

632

633

634

635

636

637

638

639

640

641

642

643

644

645

646

647

648

649

650

651

$\mathrm{AD}$ and RU designed and conceptualized the OASIS prototype, BD and PD generated iPSCs, embryonic bodies and brain organoids, $\mathrm{AD}, \mathrm{BD}, \mathrm{IR}, \mathrm{CR}, \mathrm{MB}$ and $\mathrm{MO}$ performed experiments, $\mathrm{MO}$ wrote the paper with contributions from all authors.

\section{Acknowledgements}

The authors thank Dr Elke Schmidt for help in setting up early experiments, Patrice Jegouzo (fine mechanics and 3-D printing) as well as Claire Mader and her team (CNRS FR3636 Saints Pères Central Animal Facility) for expert technical support. Confocal imaging was done at the SCM core facility (Service Commun de Microscopie, with the support of Jennifer Corridon, Paris Descartes). We thank Dr. Christian Seebacher (TILL.id) for ZEMAXing and optimizing the optical design.

Financed by the European Union (H2020 Eureka! EUROSTARS project 'OASIS', to RU and $\mathrm{MO}$ ), the CNRS (DEFI Instrumentation aux Limites, to MO), the Agence Nationale de la Recherche (ANR-10-INSB-04-01, grands investissements France-BioImaging, FBI, to MO) and the Region Ile-de-France (DIM cancéropôle, project EDISON, to MO).

The Oheim lab is a member of the C'nano IdF and Ecole de Neurosciences de Paris (ENP) excellence clusters for nanobiotechnology and neurosciences, respectively.

\section{REFERENCES}

[1] Rakotoson, I. Validation d'une approche de microscopie optique pour l'étude morphofonctionnelle des corps embryoüdes et mini-brains issus d'hIPSC, Faculté des Sciences Fondamentales et Biomédicales, Master of Science, Université Paris Descartes, (2018), 33 pages.

[2] Gonzalez, D., Gregory, J., and Brennand, K.J. (2017) The Importance of Non-Neuronal Cell Types in hiPSC-Based Disease Modeling \& Drug Screening. Front. Cell Dev. Biol. 5: 117.

[3] Fatehullah, A., Tan, S.H., and Barker, N. (2016) Organoids as an in vitro model of human development and disease. Nat. Cell Biol. 18: 246.

[4] Giandomenico, S.L., and Lancaster, M.A. (2017) Probing human brain evolution and development in organoids. Curr. Op. Cell Biol. 44: 36-43.

[5] Lancaster, M.A., Renner, M., Martin, C.-A., Wenzel, D., Bicknell, L.S., Hurles, M.E., Homfray, T., Penninger, J.M., Jackson, A.P., and Knoblich, J.A. (2013) Cerebral organoids model human brain development and microcephaly. Nature 501373. 
652 [6] Kelava, I., and Lancaster, M.A. (2016) Dishing out mini-brains: current progress and

653

654

655

656

657

658

659

660

661

662

663

664

665

666

667

668

669

670

671

672

673

674

675

676

677

678

679

680

681

682

683 future prospects in brain organoid research. Dev. Biol. 420: 199-209.

[7] Mansour, A.A., Gonçalves, J.T., Bloyd, C.W., Li, H., Fernandes, S., Quang, D., Johnston, S., Parylak, S.L., Jin, X., and Gage, F.H. (2018) An in vivo model of functional and vascularized human brain organoids. Nat. Biotech. 36: 432.

[8] Wan, P., Zhu, J., Xu, J., Li, Y., Yu, T., and Zhu, D. (2018) Evaluation of seven optical clearing methods in mouse brain. Neurophotonics 5: 035007.

[9] Renner, M., Lancaster, M.A., Bian, S., Choi, H., Ku, T., Peer, A., Chung K., and Knoblich, J.A. (2017) Self-organized developmental patterning and differentiation in cerebral organoids. EMBO J. e201694700.

[10] Hama, H., Hioki, H., Namiki, K., Hoshida, T., Kurokawa, H., Ishidate, F., Kaneko, T., Akagi, T., Saito, T., and Saido, T. (2015) ScaleS: an optical clearing palette for biological imaging. Nat. Neurosci. 18: 1518.

[11] Huisken, J., Swoger, J., Del Bene, F., Wittbrodt, J., and Stelzer, E.H.K. (2004) Optical sectioning deep inside live embryos by selective plane illumination microscopy. Science 305: 1007-1009.

[12] Keller, P.J., Schmidt, A.D., Wittbrodt, J., and Stelzer, E.H.K. (2008) Reconstruction of zebrafish early embryonic development by scanned light sheet microscopy. Science 322: 1065-1069.

[13] Santi, P.A. (2011) Light sheet fluorescence microscopy: a review. J. Histochem. Cytochem. 59: 129-138.

[14] Stelzer, E.H.K. (2015) Light-sheet fluorescence microscopy for quantitative biology. Nat. Methods 12: 23-26.

[15] Truong, T.V., Supatto, W., Koos, D.S., Choi, J.M., and Fraser, S.E. (2011) Deep and fast live imaging with two-photon scanned light-sheet microscopy. Nat. Methods 8: 757.

[16] Mahou, P., Vermot, J., Beaurepaire, E., and Supatto, W. (2014) Multicolor two-photon light-sheet microscopy. Nat. Methods 11: 600.

[17] Baumgart, E., and Kubitscheck, U. (2012) Scanned light sheet microscopy with confocal slit detection. Opt. Express 20: 21805-21814.

[18] Vettenburg, T., Dalgarno, H.I., Nylk, J., Coll-Lladó, C., Ferrier, D.E., Čižmár, T., GunnMoore, F.J., and Dholakia, K. (2014) Light-sheet microscopy using an Airy beam. Nat. Methods 11: 541. 
684 [19] Fahrbach, F.O., Gurchenkov, V., Alessandri, K., Nassoy, P., and Rohrbach, A. (2013)

685

686

687

688

689

690

691

692

693

694

695

696

697

698

699

700

701

702

703

704

705

706

707

708

709

710

711

712

713

714

715

716

717

Light-sheet microscopy in thick media using scanned Bessel beams and two-photon fluorescence excitation. Opt. Express 21: 13824-13839.

[20] Zhao, M. Zhang, H., Li, Y,. Ashok, A., Liang, R., Zhou, W., and Peng, L. (2014) Cellular imaging of deep organ using two-photon Bessel light-sheet nonlinear structured illumination microscopy. Biomed. Opt. Express 5: 1296-1308.

[21] Müllenbroich, M.C., Turrini, L., Silvestri, L., Alterini, T., Gheisari, A., Vanzi, F., Sacconi, L., and Pavone, F.S. (2018) Bessel beam illumination reduces random and systematic errors in quantitative functional studies using light-sheet microscopy. Front. Cell. Neurosci. 12: 315.

[22] Andilla, J., Jorand, R., Olarte, O.E., Dufour, A.C., Cazales, M., Montagner, Y.L., Ceolato, R., Riviere, N., Olivo-Marin, J.-C., and Loza-Alvarez, P. (2017) Imaging tissue-mimic with light sheet microscopy: A comparative guideline. Sci. Rep. 7: 44939.

[23] Elisa, Z., Toon, B., De Smedt, S.C., Katrien, R., Kristiaan, N., and Kevin, B. (2018) Technical implementations of light sheet microscopy. Microsc. Res. Tech. early view https://doi.org/10.1002/jemt.22981.

[24] Holekamp, T.F., Turaga, D., and Holy, T.E. (2008) Fast three-dimensional fluorescence imaging of activity in neural populations by objective-coupled planar illumination microscopy. Neuron 57: 661-672.

[25] McGorty, R., Liu, H., Kamiyama, D., Dong, Z., Guo, S., and Huang, B. (2015) Open-top selective plane illumination microscope for conventionally mounted specimens. Opt. Express 23: 16142-16153.

[26] Chen, B.-C., Legant, W.R., Wang, K., Shao, L., Milkie, D.E., Davidson, M.W., Janetopoulos, C., Wu, X.S., Hammer, J.A., and Liu, Z. (2014) Lattice light-sheet microscopy: imaging molecules to embryos at high spatiotemporal resolution. Science 346: 1257998 .

[27] Reynaud, E.G., Peychl, J., Huisken, J., and Tomancak, P. (2014) Guide to light-sheet microscopy for adventurous biologists. Nat. Methods 12: 30 .

[28] Oheim, M., Beaurepaire, E. Chaigneau, E., Mertz, J., and Charpak, S. (2001) Twophoton microscopy in brain tissue: parameters influencing the imaging depth. $J$. Neurosci. Meth. 111: 29-37.

[29] Bewersdorf, J., Pick, R., and Hell, S.W. (1998) Multifocal multiphoton microscopy. Opt. Lett. 23: 655-657. 
718 [30] Egner, A., Andresen, V., and Hell, S.W. (2002) Comparison of the axial resolution of 719 practical Nipkow - disk confocal fluorescence microscopy with that of multifocal multiphoton microscopy: Theory and experiment. J. Microsc. 206: 24-32.

721 [31] Shimozawa, K.Y., Kondo, T., Hayashi, S., Shitamukai, A., Konno, D., Matsuzaki, F., 722 Takayama, J., Onami, S., Nakayama, H., and Kosugi, Y. (2013) Improving spinning disk confocal microscopy by preventing pinhole cross-talk for intravital imaging.

725

726

727

728

729

730

731

732

733

734

735

736

737

738

739

740

741

742

743

744

745

746

747

748

749

750 Proc. Natl. Acad. Sci. USA 110: 6241.

[32] König, K., Multiphoton Microscopy and Fluorescence Lifetime Imaging: Applications in Biology and Medicine, Walter de Gruyter GmbH \& Co KG, 2018.

[33] Bink, K., Walch, A., Feuchtinger, A., Eisenmann, H., Hutzler, P., Höfler, H., and Werner, M. (2001) TO-PRO-3 is an optimal fluorescent dye for nuclear counterstaining in dual-colour FISH on paraffin sections. Histochem. Cell. Biol. 115: 293-299.

[34] Prieto, D., Aparicio, G., Morande, P.E., and Zolessi, F.R. (2014) A fast, low cost, and highly efficient fluorescent DNA labeling method using methyl green. Histochem Cell Biol. 142: 335-345.

[35] Smith, P.G., Baldacchini, T., Carter, J., and Zadoyan, R. (2012) Two-photon microscopy/mutlimodal imaging: Femtosecond laser developments advance two-photon imaging. BioOptics World 5. http://www.bioopticsworld.com/articles/print/volume5/issue-04/features/femtosecond-laser-developments-advance-two-photonimaging.html.

[36] Ricard, C., Arroyo, E.D., He, C. X., Portera-Cailliau, C., Lepousez, G., Canepari, M., and Fiole, D. (2018) Two-photon probes for in vivo multicolor microscopy of the structure and signals of brain cells. Brain Structure and Function 223: 3011-3043.

[37] Schmidt, E.M., and Oheim, M. (2018) Two-photon imaging induces brain heating and calcium microdomain hyper-activity in cortical astrocytes. bioRxiv 321091.

[38] Richardson, D.S., and Lichtman, J.W. Clarifying tissue clearing. Cell 162: 246-257.

[39] Aoyagi, Y., Kawakami, R., Osanai, H., Hibi, T., and Nemoto, T. (2015) A rapid optical clearing protocol using 2, 2-thiodiethanol for microscopic observation of fixed mouse brain. PloS One 10: e0116280.

[40] Kuwajima, T., Sitko, A. A., Bhansali, P., Jurgens, C., Guido, W., and Mason, C. (2013) Clear $^{\mathrm{T}}$ : a detergent-and solvent-free clearing method for neuronal and non-neuronal tissue. Development 140: 1364-1368. 
751

752

753

754

755

756

757

758

759

760

761

762

763

764

765

766

767

768

769

770

771

772

773

774

775

776

777

778

779

780

781

782

783

784

785

[41] Yu, T., Zhu, J., Li, Y., Ma, Y., Wang, J., Cheng, X., Jin, S., Sun, Q., Li, X., and Gong, H. (2018) RTF: a rapid and versatile tissue optical clearing method. Sci. Rep. 8: 1964.

[42] Ying, Q.-L., and Smith, A.G. (2003) Defined conditions for neural commitment and differentiation. Meth. Enzymol. 365: 327-341.

[43] Lancaster, M.A., and Knoblich, J.A. (2014) Generation of cerebral organoids from human pluripotent stem cells. Nat. Protoc. 9: 2329.

[44] Farahany, N., Greely, H., Hyman, S., Koch, C., Grady, C., Pa $\square$ ca, S., Sestan, N., Arlotta, P., Bernat, J., and Ting, J. (2018) The ethics of experimenting with human brain tissue. Nature 556: 429.

[45] Kino, G.S. Intermediate optics in Nipkow disk microscopes. in: Pawley, J.B. (Ed.), Handbook of Biological Confocal Microscopy, 1995, pp. 155-165.

[46] Schindelin, J., Arganda-Carreras, I., Frise, E., Kaynig, V., Longair, M., Pietzsch, T., Preibisch, S., Rueden, C., Saalfeld, S., and Schmid, B. (2012) Fiji: an open-source platform for biological-image analysis. Nat. Methods 9: 676.

\section{FIGURE LEGENDS}

Fig.1 Layout and performance of the OASIS two-photon microscope. A. Simplified optical path of the custom 2P virtual light-sheet microscope. Red: IR excitation, green: fluorescence. Ti:Sapph - fs-pulsed IR laser; DS - DeepSee; BE - beam expander; $\pi$-shaper - optical element that converts the Gaussian beam into a top hat profile; $\mathrm{M}$ - mirrors; dic - dichroic mirrors; SD - spinning disk; TL - tube lens; CDE - corrective distance element; OL - objective lens; ISD - image-splitting device; sCMOS - camera. Inset o: generated multi-spot excitation pattern und epi-collection of the generated fluorescence through the same objective. Inset 2: detail of microlens/pinhole/dichroic coating arrangement. Note the offset between the excitation (exc.) and fluorescence foci (fluo.) at the level of the disk, produced by the CDE. B. Depth penetration in turbid samples. Log-plot of 2P-excited fluorescence from a green-fluorescent Chroma test slide, topped either with water $(0 \%)$ or increasing concentrations of milk (a model for the multi-scale scatterers present in tissue), for the OASIS (red dots) and a ZEISS LSM710. Confocal pinhole diameters were 1 and 2 Airy, as indicated. C. OASIS prototype, note the compact size and space available around the objective, inset. VCD - voice coil $z$ drive; BFC - bright-field camera. D. OASIS lateral resolution. Equatorial section through an autofluorescent thorny pollen grain. Scale bar, $10 \mu \mathrm{m}$. Inset: magnified 
786 view of an individual spine and intensity profiles across the lines shown; Scale bar, $2 \mu \mathrm{m}$. E. 787 OASIS $z$-resolution. Axial-intensity profile measured from a $z$-stack of images acquired from 788 a green fluorescent Chroma slide (solid line), and its derivative $d F / d z$ (dashed). The FWHM, 789 equiva-lent to the $10-90 \%$ intensity range, was taken as axial optical sectioning capacity $\Delta z$.

790 Fig. 2 Comparison of the used nuclear stains and clearing methods. A. Left, optical section of 791 a slice from an E14.5 embryo, labeled with TO-PRO3 or Methyl Green and observed on a 792 confocal laser scanning microscope (CLSM, top) or on the OASIS microscope (bottom). 793 Scale-bar, $10 \mu \mathrm{m}$. Right, relative fluorescence intensity of TO-PRO3 and Methyl Green in 794 nuclei of E14.5 embryo slices measured on a CLSM (top right) or on the OASIS (bottom 795 right). B. TO-PRO3 (top) and Methyl Green (bottom) 2P-excitation spectra. Color code as in 796 (A). C. Macrophotographies of E10.5 embryos in control (PBS) and following three clearing 797 protocols (Clear ${ }^{\mathrm{T} 2}, \mathrm{RTF}$ and TDE 60\%). Note the variable degree of transparisation and the 798 volume change. D. Relative fluorescence of TO-PRO3-positiev nuclei in E14.5 embryo slices 799 observed on a CLSM after the same clearing protocols as above. Note the log-scale. ${ }^{*}=P<$ 800 0.0001. E. Fluorescence loss upon clearing requires high laser powers. Confocal micrographs 801 of slices from an E14.5 embryo labeled with TO-PRO3 under control (left) and after RTF 802 clearing (right) along with the laser powers required to attain the same signal-to-background 803 ratio. Scale-bar, $10 \mu \mathrm{m}$.

804

805 Fig. 3 The OASIS microscope outperforms a CLSM for 3-D embryo imaging. A. 3-D data set 806 taken with the OASIS in a non-cleared embryo stained with TO-PRO3 (Control). Panels show $807 x z$-projection of a $z$-stack of images (right) and $x y$-planes (left) corresponding to the dashed 808 lines at $30 \mu \mathrm{m}$ (1) and $65 \mu \mathrm{m}$ imaging depth (2), respectively. Scale-bar, $25 \mu \mathrm{m}$. B. same, at $80932 \mu \mathrm{m}$ (1) and $115 \mu \mathrm{m}$ depth (2), respectively, for an RTF-cleared embryo. Scale-bar, $25 \mu \mathrm{m}$, 810 as in (A). C. Representative bleaching curves during continuous acquisitions, from a single $z$ 811 section in - respectively -a thin slice of intestine (top) and of TO-PRO3-labeled nuclei 812 (bottom) at 30- $\mu \mathrm{m}$ imaging depth in a embryo. D. Representative planes at various imaging 813 depths $(15,50$ and $100 \mu \mathrm{m}$, respectively) of a 3-D data set (200 planes from the surface to 200 $814 \mu \mathrm{m}, \Delta z=1 \mu \mathrm{m}$;) acquired from a TO-PRO3-labled embryo. The 2 colums show the sections 815 during the $1^{\text {st }}$ passage (left) and during the $2^{\text {nd }}$ passage, after completion of the $1^{\text {st }}$ image stack 816 (right). Note the almost complete bleaching prohibiting repetitive volume imaging for the 817 CSLM but not the OASIS microscope. Measured laser powers are given for each depth. 818 Scale-bar, $25 \mu \mathrm{m}$. 
820 Fig. 4 3-D imaging with the OASIS of the fine structure of embryonic bodies (EBs). A. 821 Macrophotography of a day-7 EB; Scale-bar, $300 \mu \mathrm{m}$. B. Image acquired in the center of the 822 organoid after TO-PRO3 staining, $50 \mu \mathrm{m}$ below the surface. Scale-bar, $25 \mu \mathrm{m}$. Note the 823 presence of internal round structures with a lumen. C. Zoom on mitotic figures observed in 824 the EB at various depth (top left: prophase; top right: metaphase; bottom left: early anaphase; 825 bottom right: late anaphase); stain: TO-PRO3. Scale-bar for all panels, $10 \mu \mathrm{m}$. D. $x z$-pro826 jections of a $z$-stack acquired across an entire day-7, TO-PRO3-stained EB. Scale-bar, $25 \mu \mathrm{m}$. 827 Arrow points to mitosis also visible in panel (E). \# indicates ventricle-like structure also 828 perceived in (E). E. $x y$-sections across the dotted lines shown in (D) at imaging depths of, 829 respectively, $42 \mu \mathrm{m}$ (1), $75 \mu \mathrm{m}$ (2) and $155 \mu \mathrm{m}$ (3). Scale-bar, $25 \mu \mathrm{m}$. With the exception of 830 (A), all images were acquired after RTF clearing.

831

832 Figure 5. Brain organoid imaging with OASIS. A. Macrophotography of a day 11 brain 833 organoid before clearing and observation on the OASIS microscope; scale-bar: $300 \mu \mathrm{m}$. B. 834 Image acquired in the center of the organoid at $45 \mu \mathrm{m}$ below the surface; stain: TO-PRO3; 835 scale-bar: $10 \mu \mathrm{m}$. C. z-reconstruction (left) and xy-acquisition (right) at respectively $30 \mu \mathrm{m}(1)$, $83680 \mu \mathrm{m}(2)$ and $125 \mu \mathrm{m}$ (3) below the surface of the organoid; stain: TO-PRO3; scale-bar: $83725 \mu \mathrm{m}$. Note the change of the morphology of the nuclei in the center of the region of interest 838 when depth increase. D. z-reconstruction (left) and xy-acquisition (right) at respectively $20 \mu \mathrm{m}$ 839 (1), $35 \mu \mathrm{m}(2)$ and $75 \mu \mathrm{m}$ (3) below the surface of the organoid; stain: TO-PRO3; scale-bar: $84025 \mu \mathrm{m}$. Note the globular structure (neuro-epithelium) that can also be observed on the 841 macroscopy and numerous mitosis inside this structure (arrows). Except in A. all images were 842 acquired after the clearing of the organoid with the RTF protocol. Note the image quality 843 across the entire imaged volume.

844

845

846

847 
A

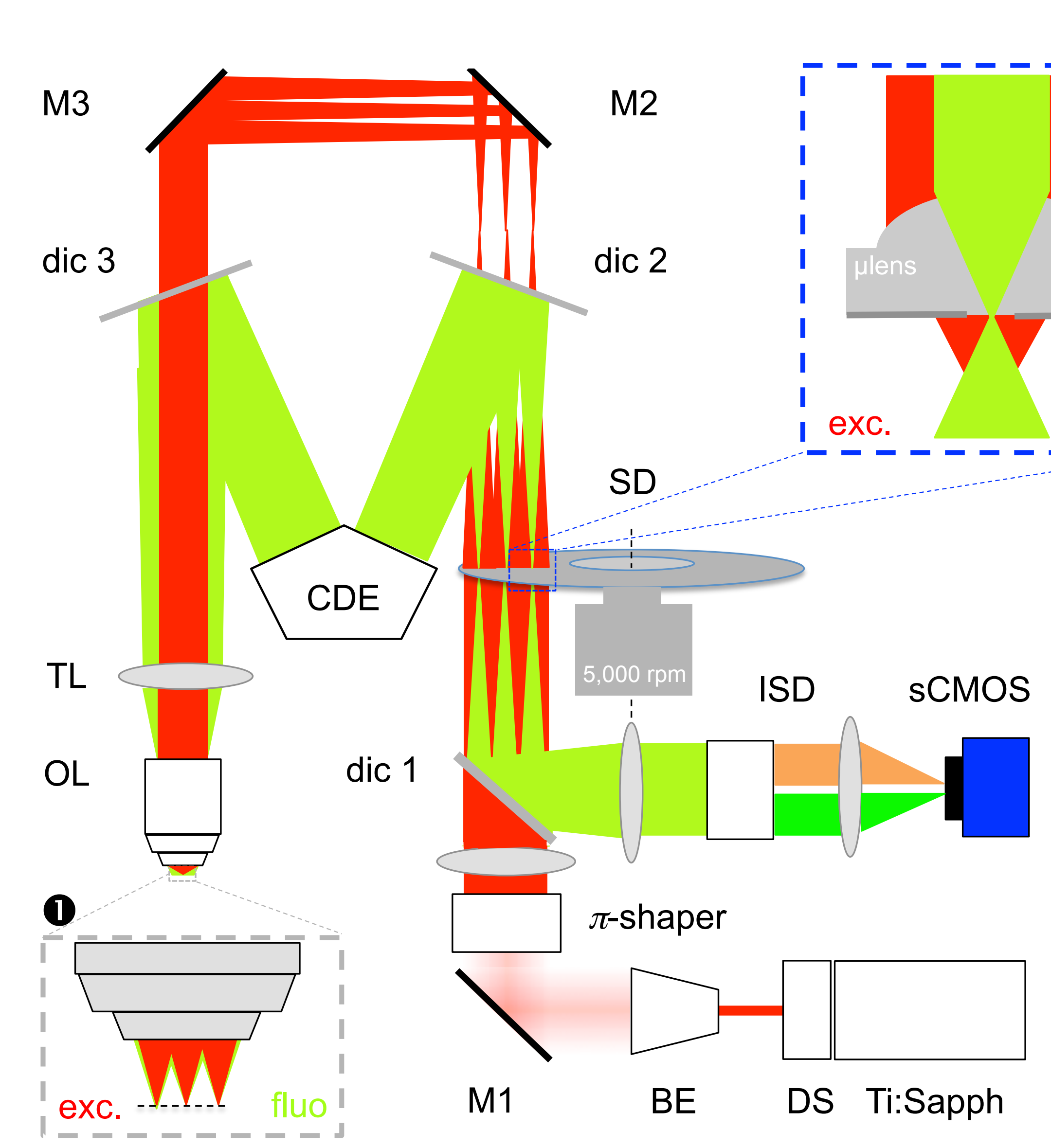

B

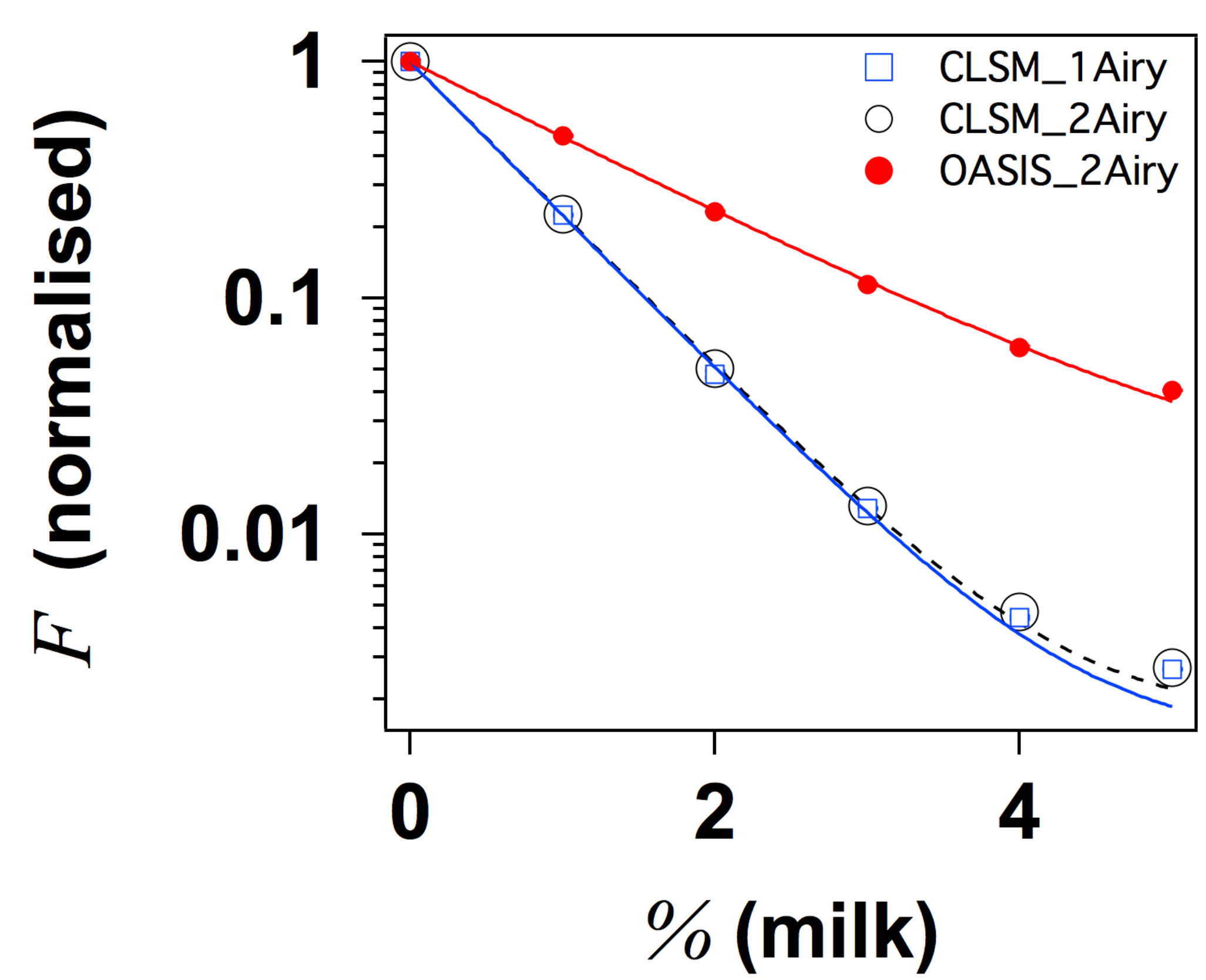

C
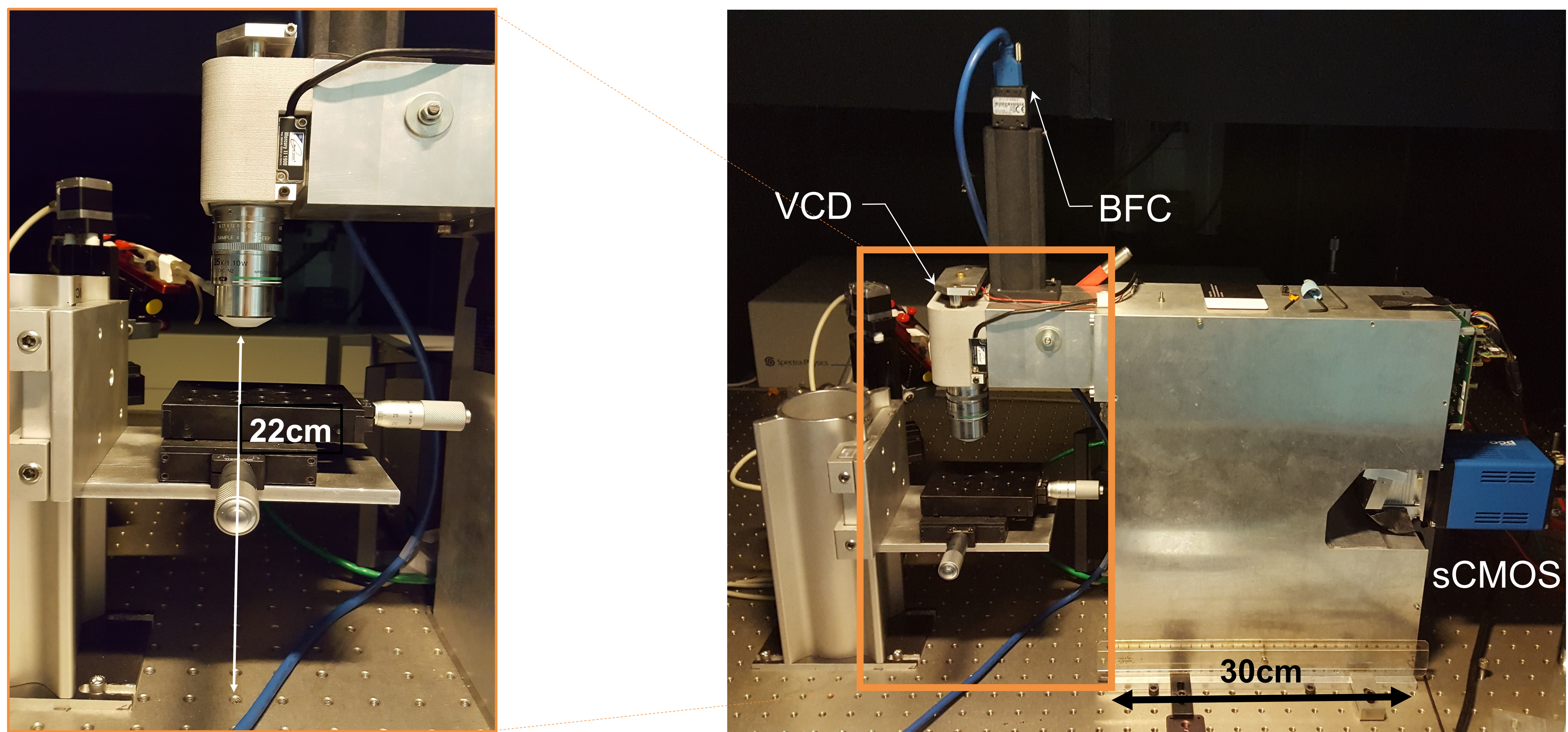

D

E

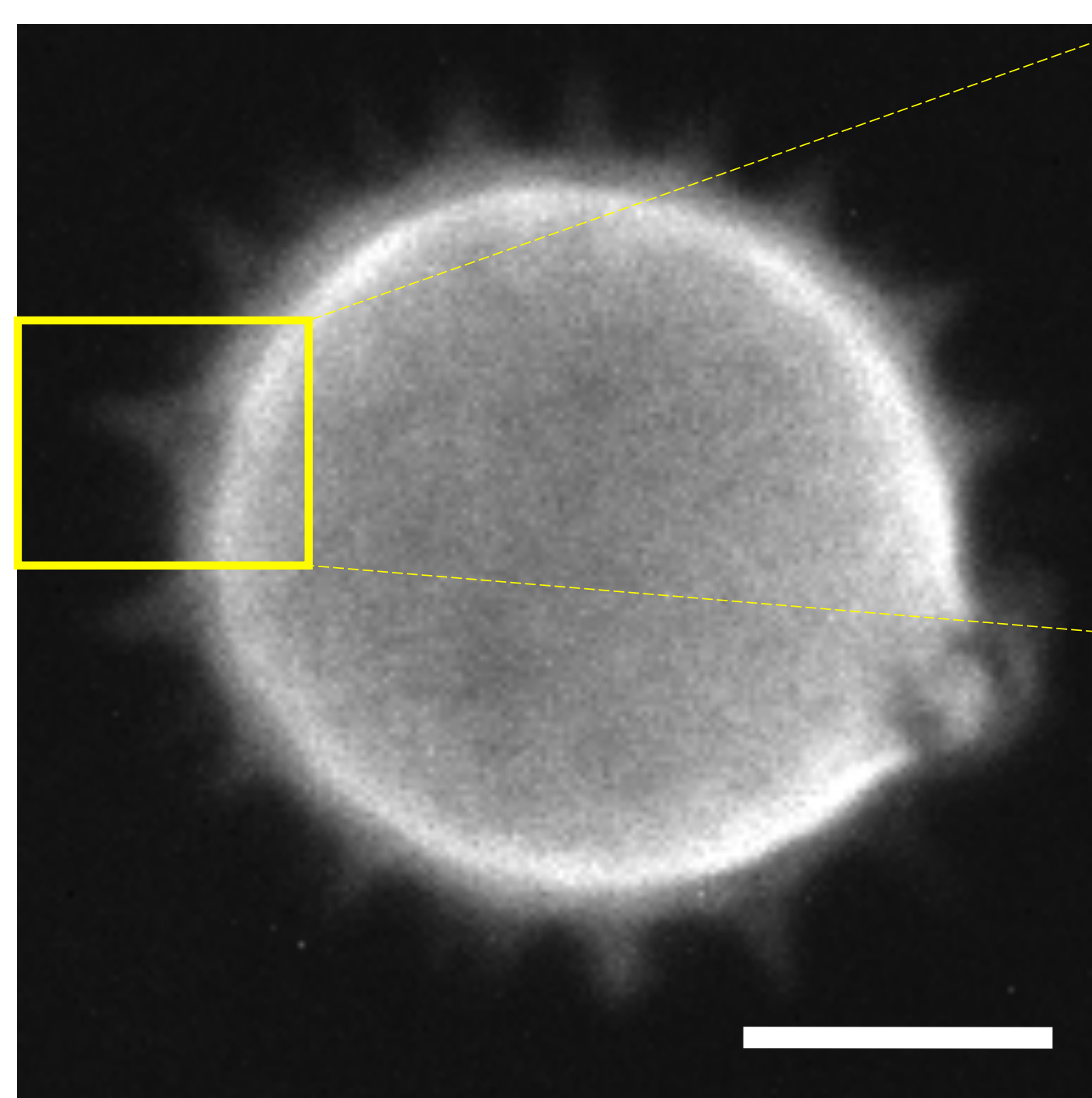

Pollen

OASIS: $43 \mathrm{~mW}$; 1,075 mW/spot ; 760nm
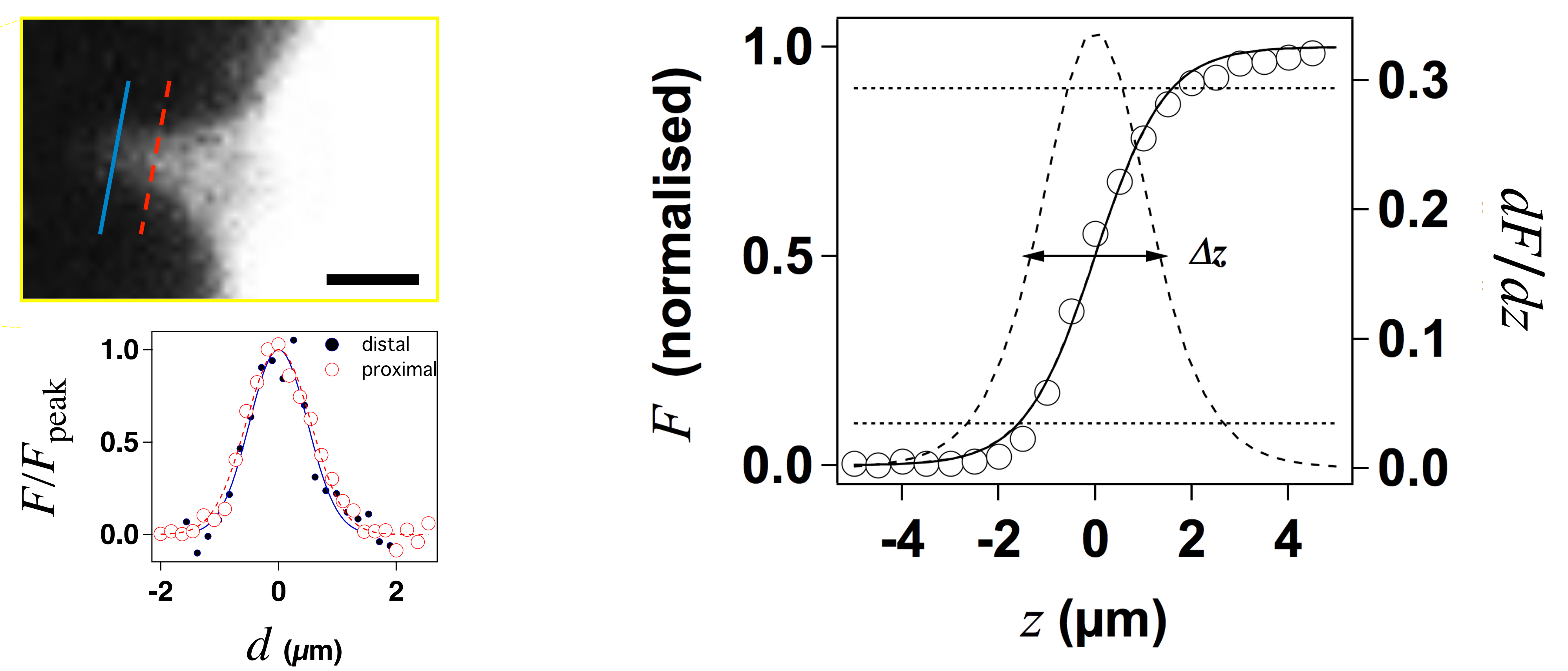

Figure 1 
A

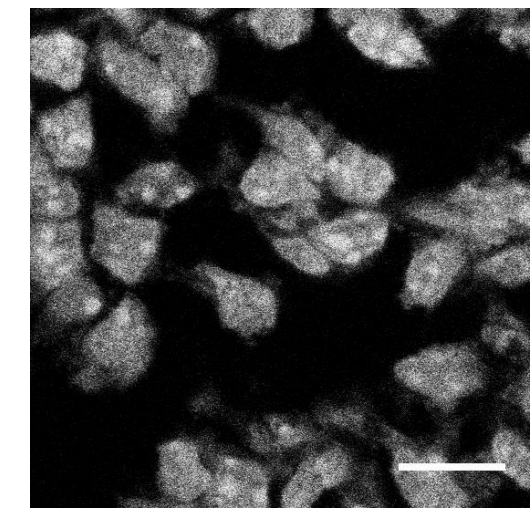

ToPro3

Confocal: $0,55 \mu \mathrm{W}-633 \mathrm{~nm}$

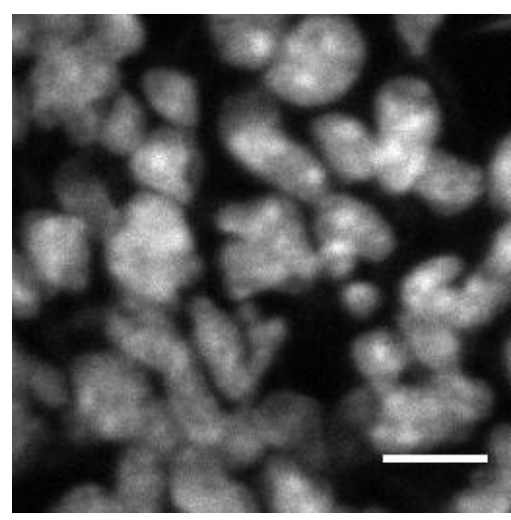

ToPro3

OASIS: $309 \mathrm{~mW}$; 7,7 mW/spot ; 760nm

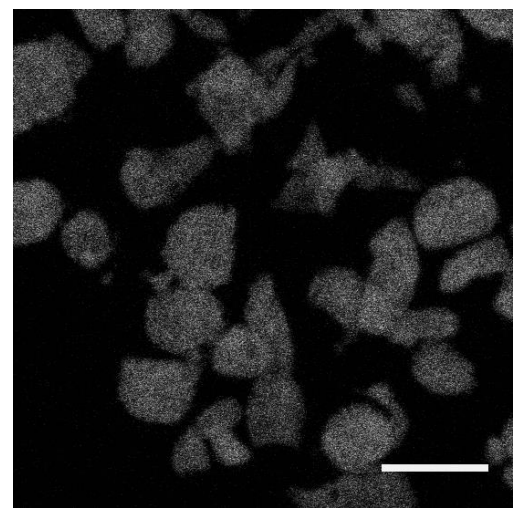

Methyl Green

Confocal: $0,55 \mu \mathrm{W}-633 \mathrm{~nm}$

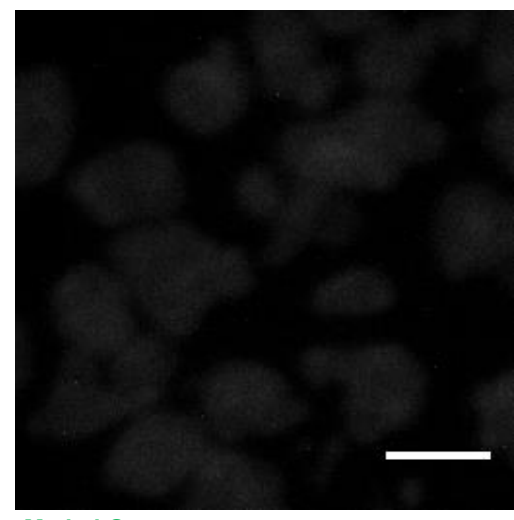

Methyl Green

OASIS: $309 \mathrm{~mW}$; 7,7 mW/spot ; 750nm
B

Fluorescence intensity at $633 \mathrm{~nm}$

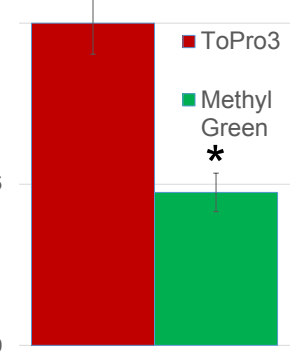

Fluorescence intensity on the OASIS

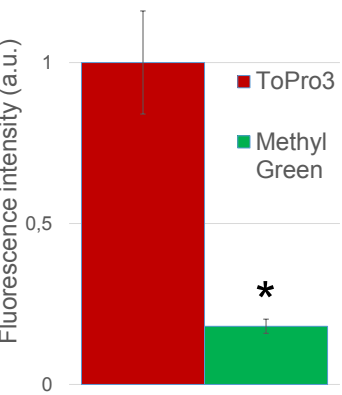

ToPro3 2hv

excitation spectrum

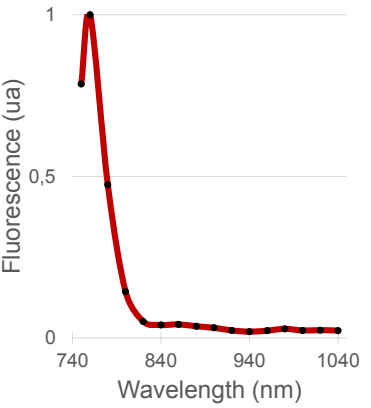

Methyl Green 2hv excitation spectrum

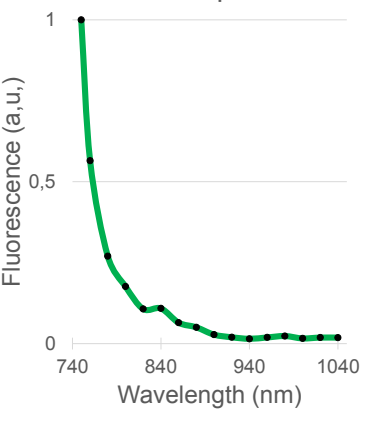

C

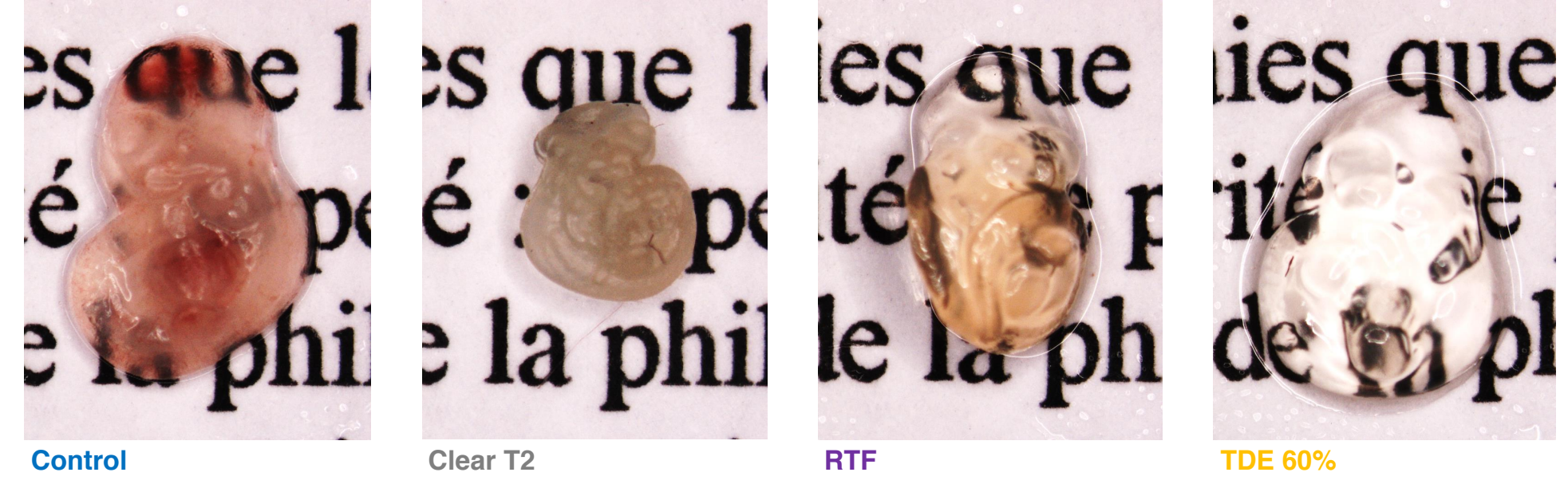

D

ToPro3 fluorescence under various clarification

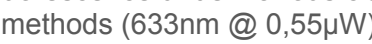

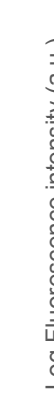

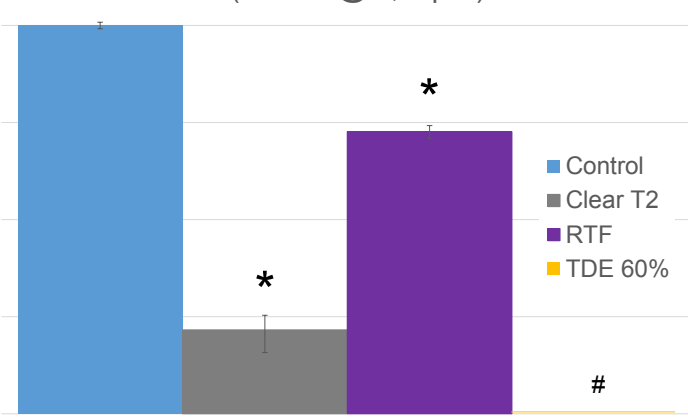

E

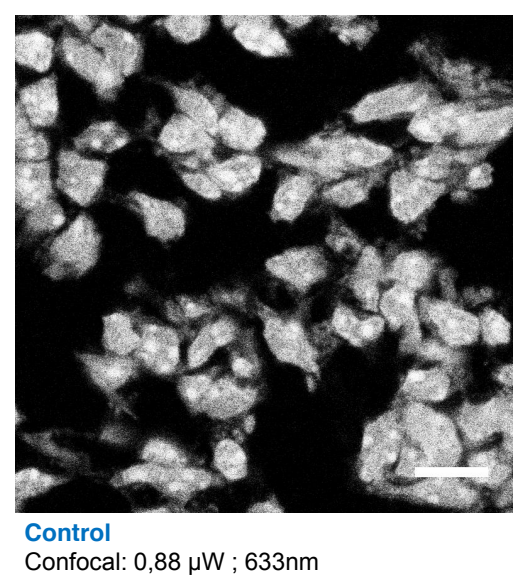

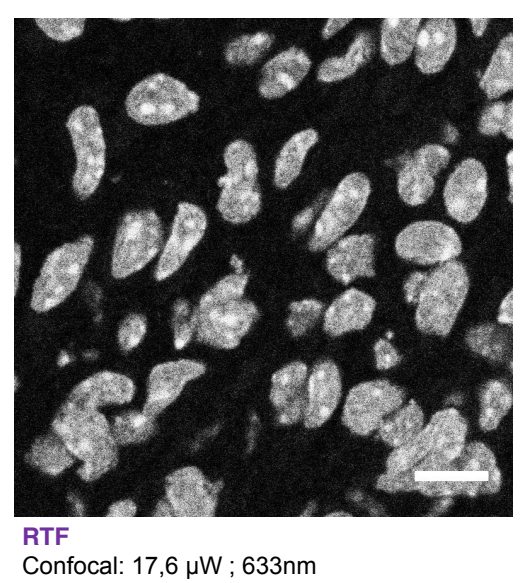

Figure 2 
A
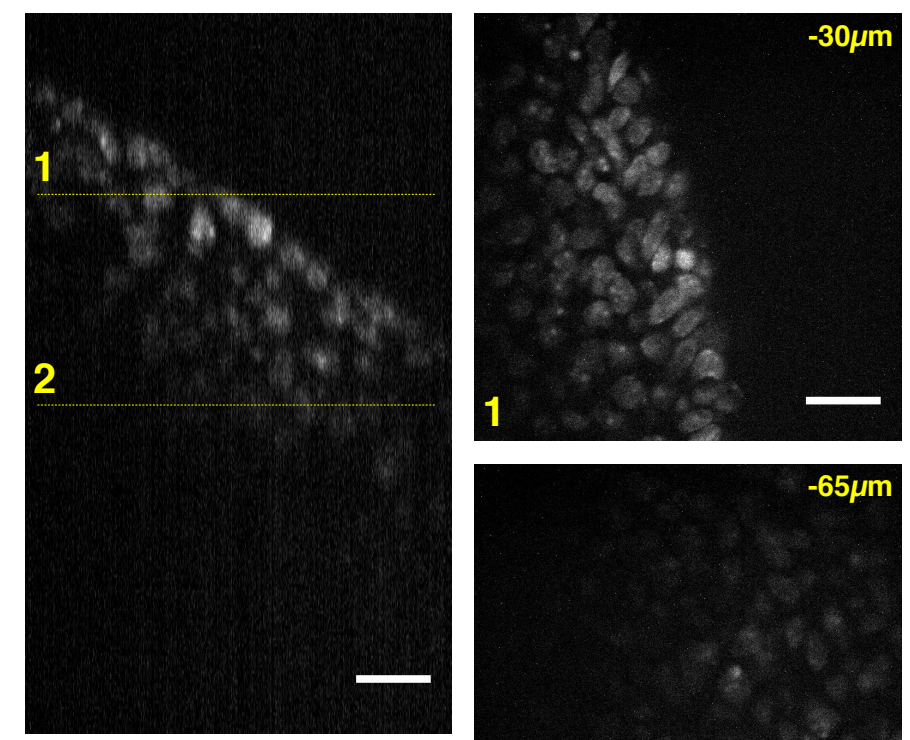

$-65 \mu \mathrm{m}$

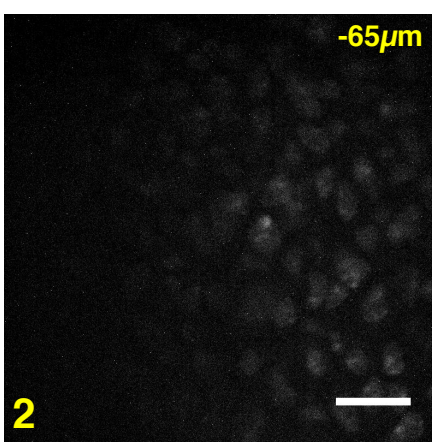

Control ( $z$ )

OASIS: $626 \mathrm{~mW}$ : $15,7 \mathrm{~mW} / \mathrm{spot}$ : $760 \mathrm{~nm}$

C
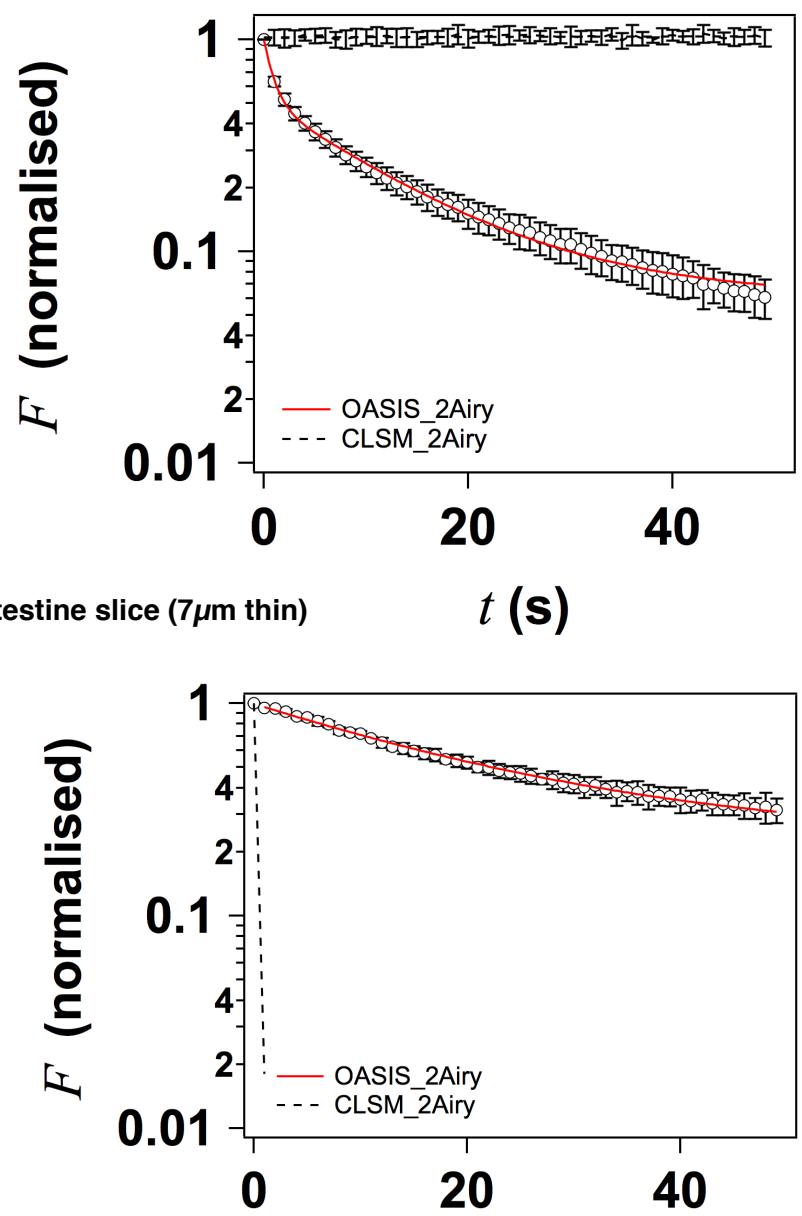

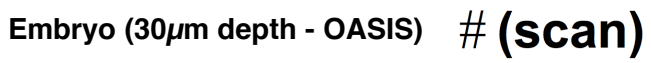

\section{Figure 3}

B

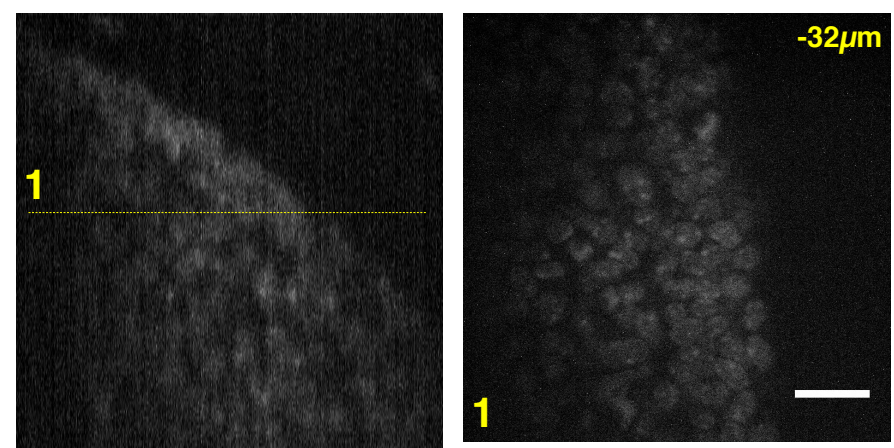

$-115 \mu \mathrm{m}$

2

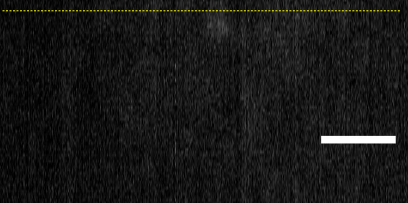

RTF $(x y)$

OASIS: $626 \mathrm{~mW} ; 15,7 \mathrm{~mW} / \mathrm{spot}$; 760nm

D
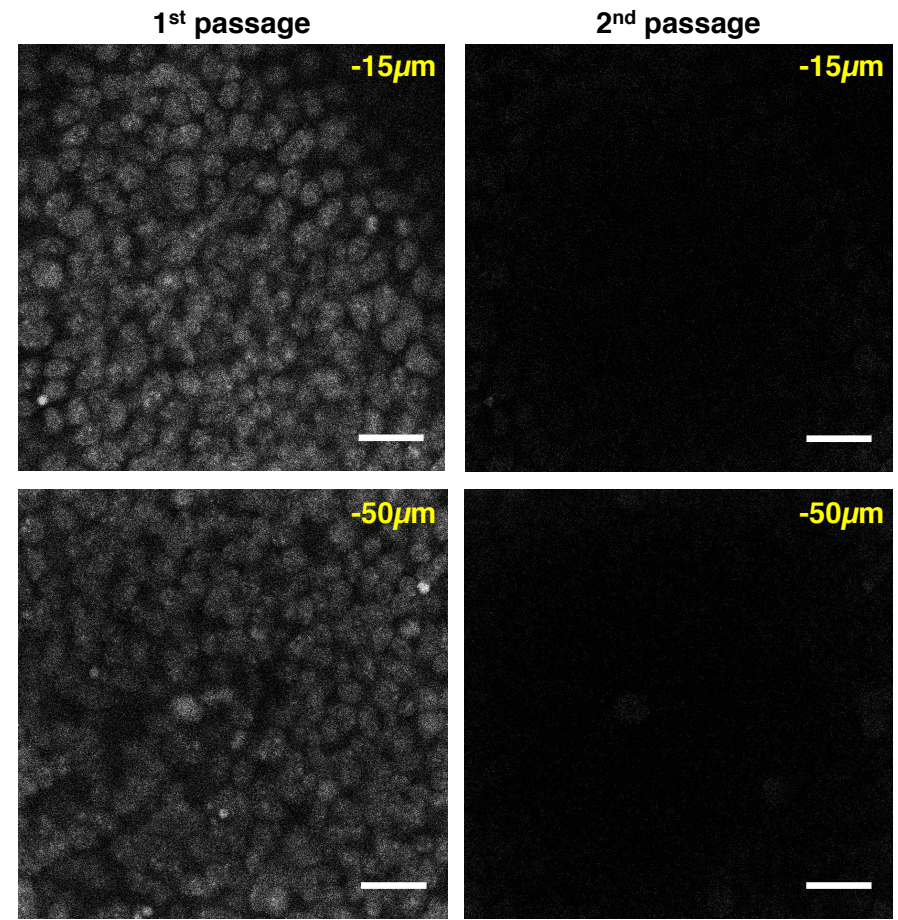

$-50 \mu \mathrm{m}$

$-100 \mu \mathrm{m}$

$-100 \mu \mathrm{m}$

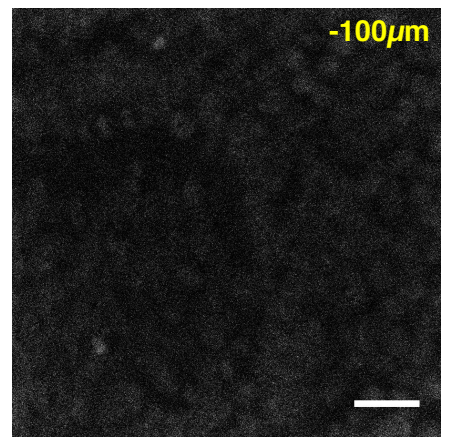

Control $(x y)$

Confocal: 9,$36 ; 15,6 ; 26 \mu \mathrm{W} ; 633 \mathrm{~nm}$
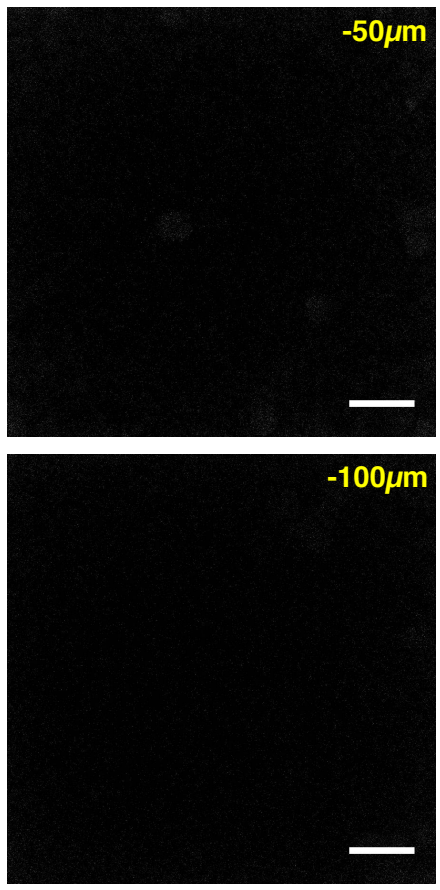
A

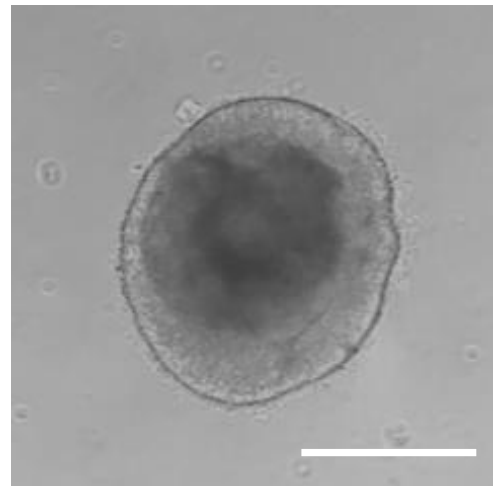

Control (xy)

Macroscope

D

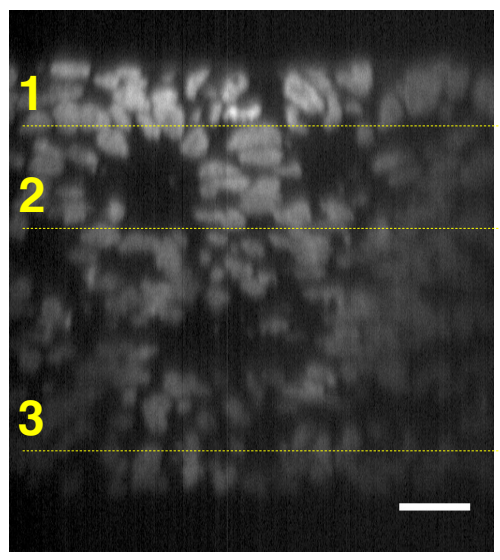

RTF ( $\mathbf{2})$

OASIS: $630 \mathrm{~mW}$; 15,7 mW/spot ; 760nm

E

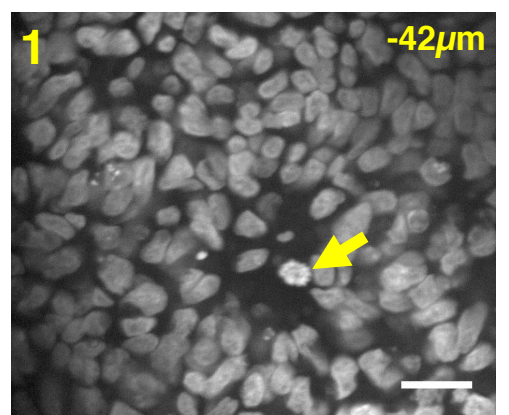

RTF (xy)

OASIS: $630 \mathrm{~mW}$; $15,7 \mathrm{~mW} / \mathrm{spot}$; 760nm
B

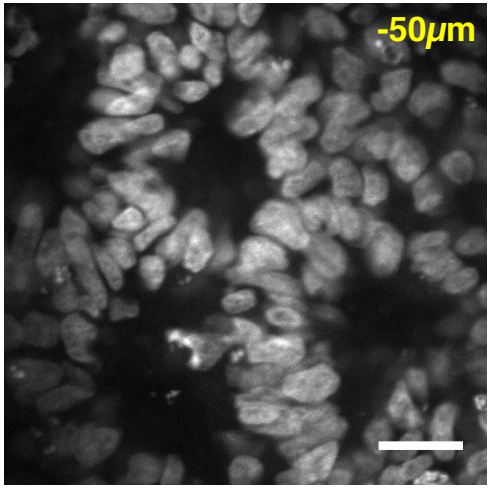

RTF (xy)

OASIS: $616 \mathrm{~mW}$; 15,4 mW/spot ; 760nm
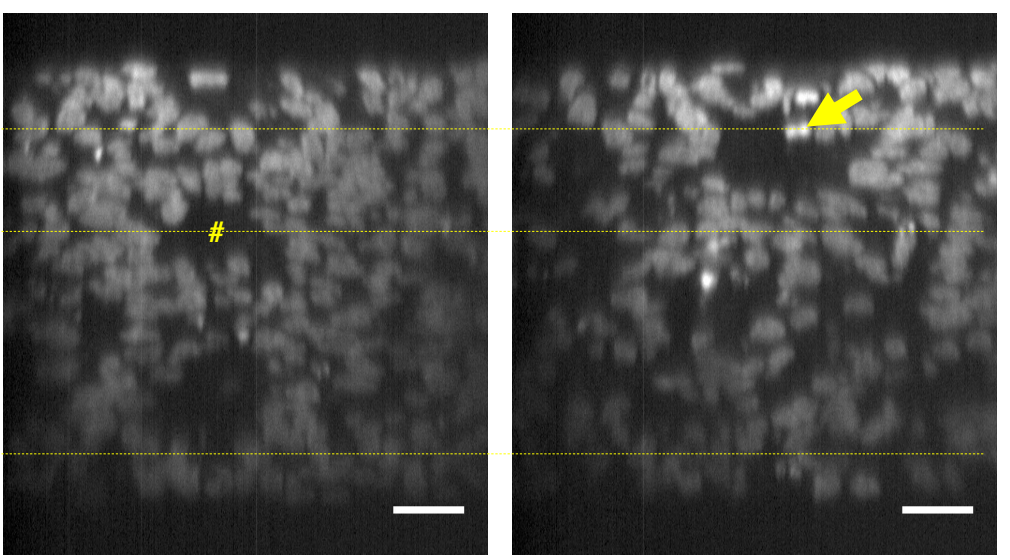

RTF (xy)

OASIS: $616 \mathrm{~mW}$; 15,4 mW/spot ; 760nm
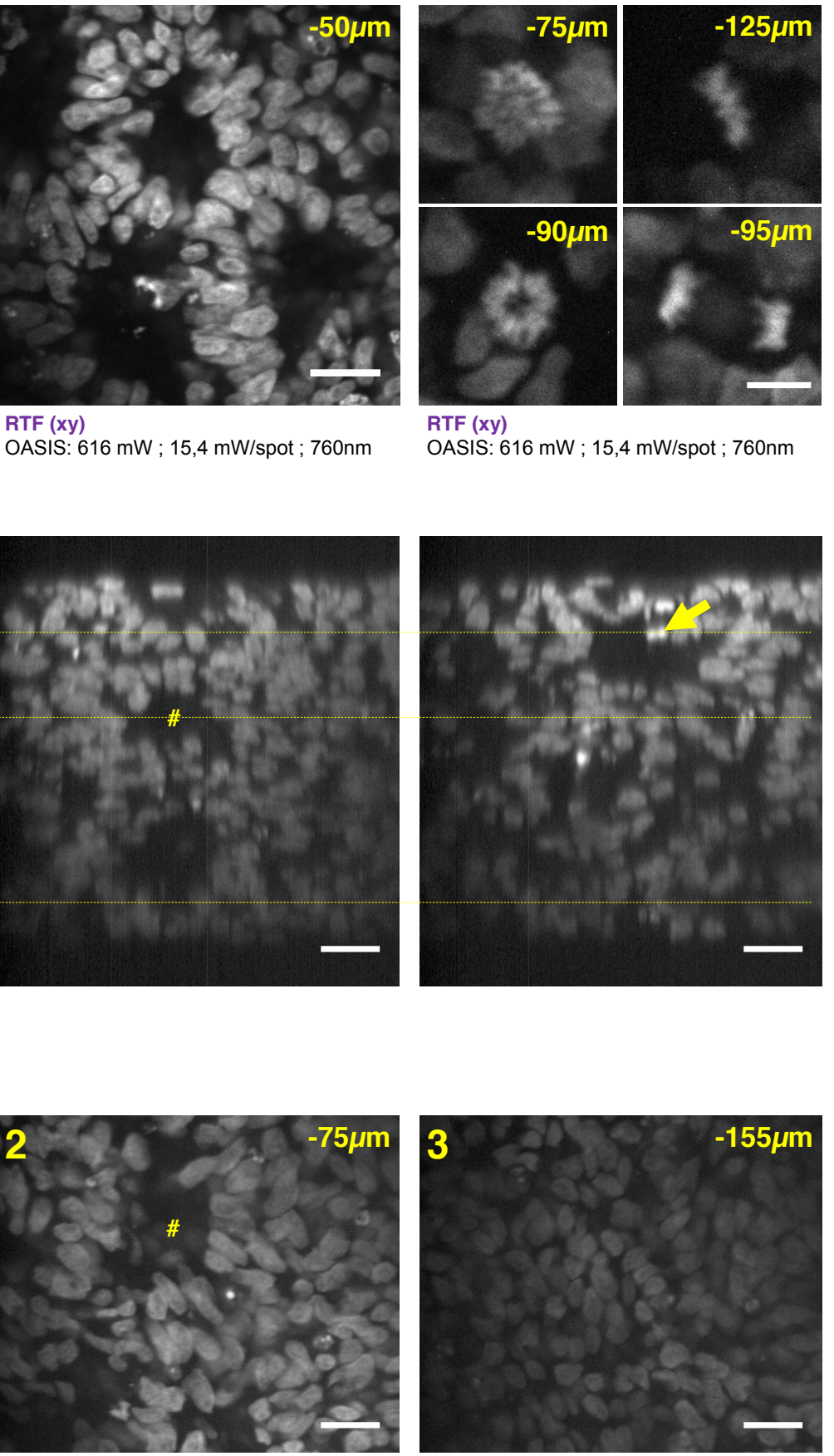

\section{Figure 4}




\section{A}

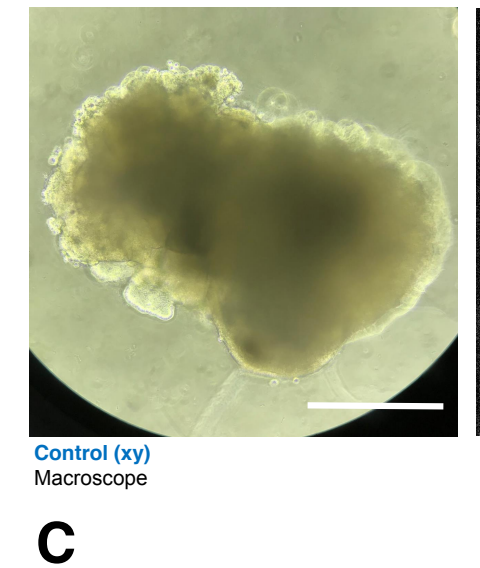

1

2

3

OASIS: $614 \mathrm{~mW}$; 15,4 mW/spot ; 760nm

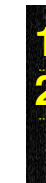

D

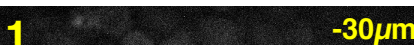

$-30 \mu \mathrm{m}$
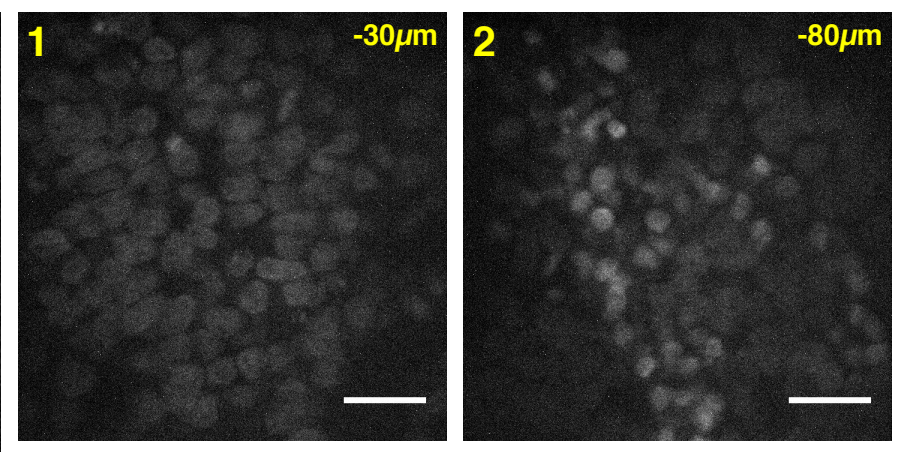

3

$-125 \mu \mathrm{m}$

\section{C8}

2

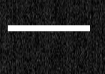

RTF (z)

Figure 5

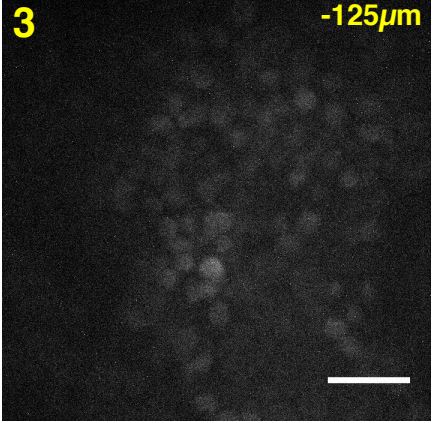

3

$-75 \mu \mathrm{m}$

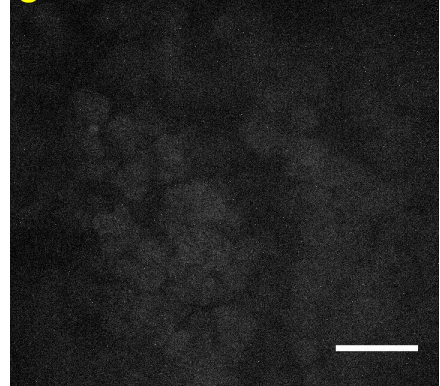

RTF (z)
OASIS: $614 \mathrm{~mW}$; 15,4 mW/spot ; 760nm
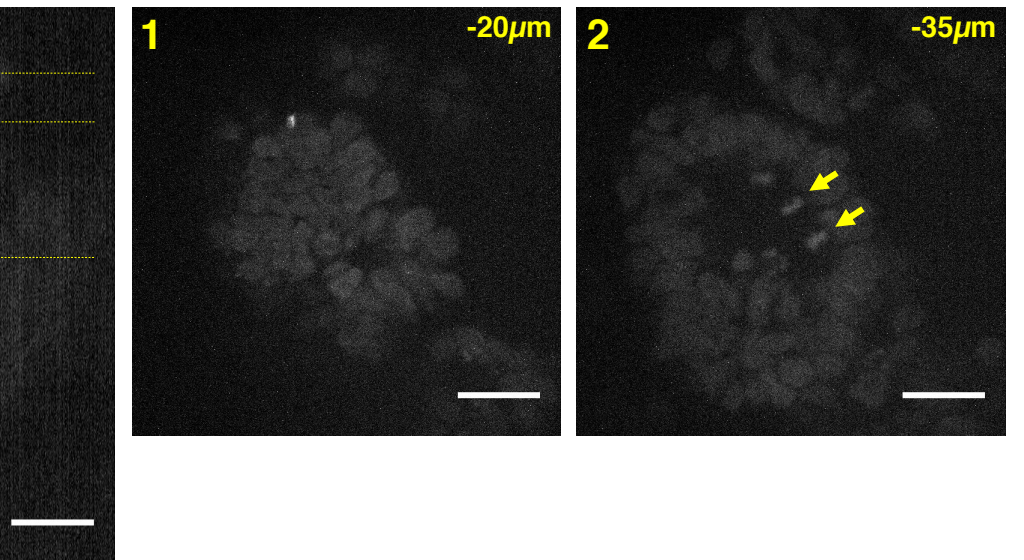

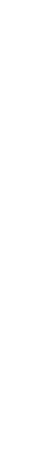

